\title{
Mapping the expansion of the Northwest Magdalenian
}

\author{
Rebecca Miller \\ Université de Liège, Service de Préhistoire 7, place du XX août, bât. A1, B-4000 Liège, Belgium
}

\section{A R T I C L E I N F O}

\section{Article history:}

Available online 30 May 2012

\begin{abstract}
A B S T R A C T
New paleoclimatic data for the Lateglacial clarify climatic phases during the Lateglacial. Cold climate played a key role in limiting Magdalenian expansion from southwest and central Europe This paper presents chronological and geographic data for the different climatic phases to map the expansion of the Magdalenian into northwest Europe. Specifically, it can be seen that initial expansion follows a southwest-northeast trajectory into southern Germany, followed by northern expansion into Belgium, central Germany and the Paris Basin at the end of the Oldest Dryas. During the Bølling and Allerød phases, human occupation intensified in the Paris Basin and contacts and/or territorial exploitation between both the Paris Basin and the Rhineland region with Belgian territory is demonstrated.
\end{abstract}

(c) 2012 Elsevier Ltd and INQUA. All rights reserved.

\section{Introduction}

Significant research, both in the field and the laboratory, has been carried out in Northwest Europe over the last two decades to address the arrival and establishment of the Magdalenian technocomplex in this region, focusing on different issues relating to its chronology, expansion, cultural components, settlement patterns and subsistence strategies (see e.g., Rozoy, 1988; Vermeersch and Symens, 1988; Rensink, 1993; Otte and Straus, 1997; Kozłowski, 2001; Cattelain, 2005; Street et al., 2009). More specifically, the chronology of Magdalenian expansion has been the subject of critical research on the viability of the radiocarbon and AMS dates obtained for Magdalenian sites, their context and interpretation with respect to the expansion of the Magdalenian from southern Europe. For example, Charles $(1994,1996,1998)$ undertook a critical review of available and new AMS dates for sites in the Belgian Ardennes, rejecting certain early dates and arguing for Magdalenian occupation "at the interface between Dryas I and the Bølling Interstadial phase" (Charles, 1998, p. 212) during the Bølling. The discussion between Housley et al. (1997, 2000) and Blockley et al. (2000) raises the issue of the validity of calibrating radiocarbon dates and their interpretation, giving rise to two competing hypotheses for the recolonization of Northern Europe, one of which argues for a two-stage process (Housley et al., 1997, 2000), and the other for a reduced population but not complete abandonment (Blockley et al., 2000). The present paper exploits the geographic and chronological data available in the Radiocarbon Palaeolithic Database version 12 to map the dated Magdalenian sites from the

E-mail address: rmiller@ulg.ac.be. onset of deglaciation after the Last Glacial Maximum to the end of the Magdalenian and the appearance of new technocomplexes prior to the Pleistocene-Holocene transition. The objective is to identify the broad trends of population movement during the Lateglacial using such data. It will be argued that the initial phase of expansion involved movement from the southwest to northeast, from Southwest and Central France to Southern Germany, followed by subsequent expansion northward to the Paris Basin.

\section{Lateglacial climate}

Recent paleoclimatic research using different proxies has significantly clarified the chronology and mechanisms for Lateglacial climate change (see e.g., Alley and Clark, 1999; Severinghaus and Brook, 1999; Alley et al., 2003; Clark et al., 2009; Liu et al., 2009; Shakun and Carlson, 2010; Williams et al., 2010). Based on Litt et al. (2001, Table 3, p. 1247), the onset of post-Last Glacial Maximum deglaciation occurs at 20-19 ka cal BP (Clark et al., 2009, Fig. 3, p. 711), with a phase of gradual warming from 19 to $17.5 \mathrm{ka}$ cal BP. Heinrich event 1 occurs from 17.5 to $16.5 \mathrm{ka}$ cal BP, a cold phase, followed by pre-BA (Bølling-Allerød), a warming phase, stabilizing at $14.67 \mathrm{ka}$ cal BP, the threshold between stadial and interstadial climate phases. The Bølling-Allerød Interstadial (14.67-12.9 ka cal BP) includes GRIP (INTIMATE) phases GI-1e (Meiendorf), GI-1d (Oldest Dryas), I-1c to 1a (Allerød). This is followed by GS-1 (Younger Dryas). Liu et al. (2009) present a synchronously coupled atmosphere-ocean general circulation model that simulates "abrupt BA warming as a transient response of the Atlantic meridional overturning circulation (AMOC) to a sudden termination of freshwater discharge to the North Atlantic before the BA" (Liu et al., 2009, p. 310) (Fig. 1). The Intra-Allerød 

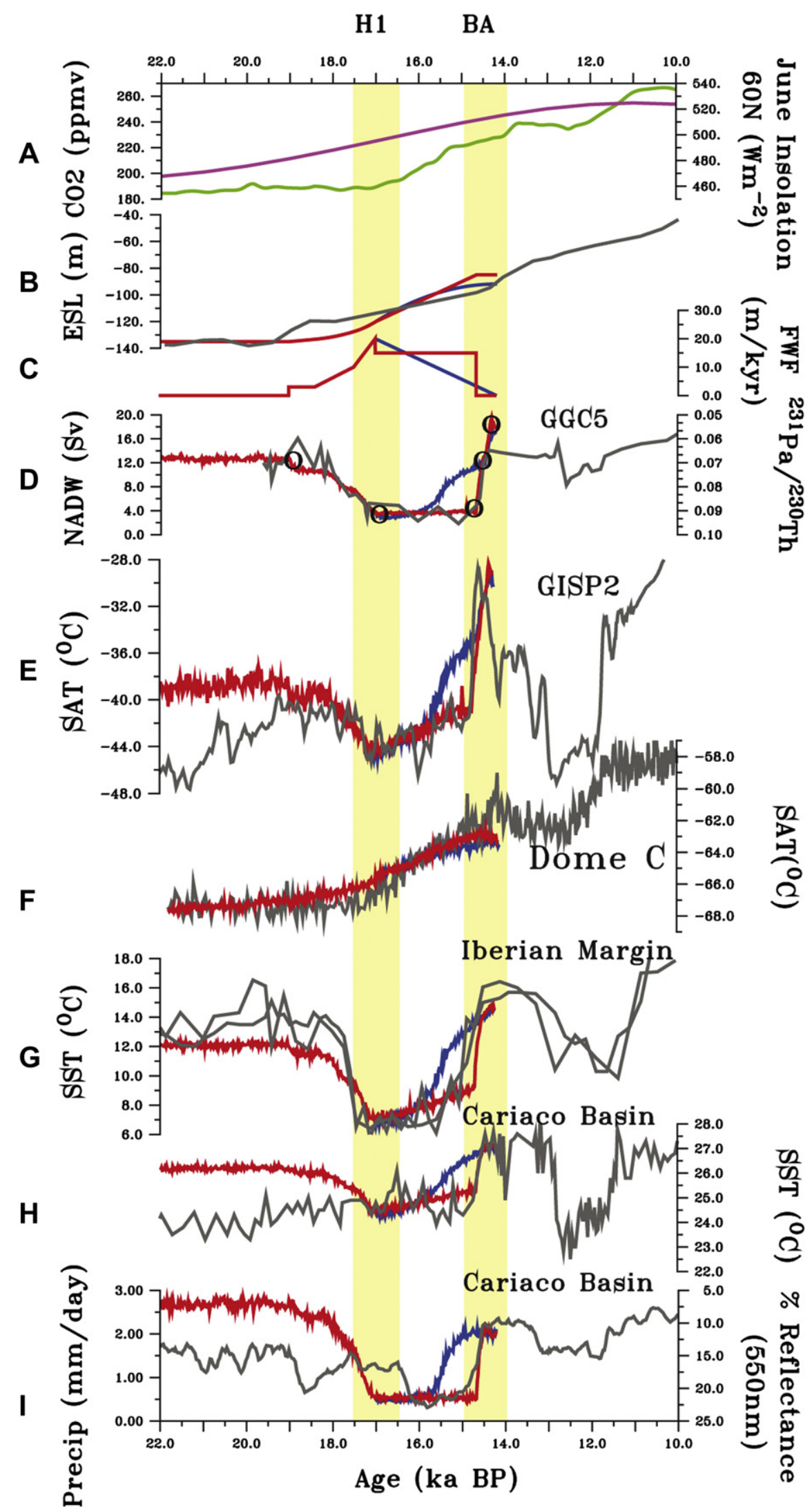

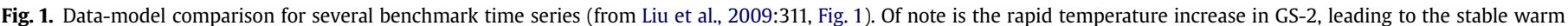
Bølling phase (GI-1e). 
Cold Period (IACP) lasting around 150 years, was likely caused by pulses of glacial meltwater to the North Atlantic (Donnelly et al., 2005, p. 89). The Allerød is followed abruptly by the Younger Dryas (12.6-11.5 ka cal BP), considered to be a Heinrich event (Severinghaus et al., 1998; Sima et al., 2004; Brauer et al., 2008), also ending suddenly with the Preboreal phase of the Holocene (11.5-10 ka cal BP) (e.g., Björck et al., 1997).

Some differences exist between this climatic schema and the "traditional" definitions, which place the Bølling in GI-1e and the Allerød in G-1c to G-1b. Climate changes occurring during the Lateglacial played a key role in limiting or permitting the expansion of Magdalenian populations, as will be seen below. The phases used here are shown in Table 1 (based on Litt et al., 2001, Table 3, p. 1247).

\section{Material and methods}

Using the dates and coordinates available in the Radiocarbon Palaeolithic Database version 12 (Vermeersch, 2011), the expansion of the Magdalenian from southwest France to northwest Europe is mapped here in relation to the climatic oscillations of the Lateglacial (Fig. 2a-h, Tables 2-9). Sites in Northwest Europe (France, Belgium, Netherlands, Germany, Switzerland, Great Britain) have been included in the analysis; sites further north or east have not. A limit at $47^{\circ} \mathrm{N}$ latitude is clearly a threshold separating the relatively dense concentration of early Lateglacial sites south of this limit from the first sites reflecting Magdalenian expansion north of this latitude. The validity of dates for sites north of $47^{\circ} \mathrm{N}$ has been evaluated for this paper. Dates for sites south of this boundary, in southwest and central France, have not been evaluated; some of these dates, most conventional, may therefore be erroneous. Given the relatively dense concentration of sites present in these regions, the elimination of a few dates would likely not change the overall pattern, but would be important rather for the distinction between contemporaneous technocomplexes or cultures such as the Magdalenian, Badegoulian and Solutrean. Such distinctions are not addressed in this paper, which focuses on sites expanding north of $47^{\circ} \mathrm{N}$. ${ }^{14} \mathrm{C}$ dates have been calibrated with OxCal v.4 to produce ages in ka cal BP to correspond to the dated climatic phases; the original uncalibrated dates are available in the Radiocarbon Palaeolithic Database version 12 as well as in the tables found in the Supplementary data. However, given advances and disadvantages in calibrating radiocarbon dates (see Street et al., 1994), the calibrated dates presented here should not be considered definitive. Time ranges reflect the main climatic phases after the LGM (Table 1). Sites with calibrated dates falling within each date range have been plotted using Google Earth (KML files for the data are available). A short discussion is presented below for each date range.

Table 1

Date ranges for paleoclimatic phases used in the text (based on Table 3; Liu et al., 2009; Litt et al., 2001; Table 3; Björck et al., 1998).

\begin{tabular}{|c|c|c|c|}
\hline $\begin{array}{l}\text { Phase } \\
\text { (ka cal BP) }\end{array}$ & $\begin{array}{l}\text { GRIP } \\
\text { (INTIMATE) }\end{array}$ & Biozones, NW Germany & Traditional phases \\
\hline $12.9-11.5$ & GI-1a GS-1 & $\begin{array}{l}\text { Final Allerød Younger } \\
\text { Dryas }\end{array}$ & Younger Dryas \\
\hline $13.9-12.9$ & $G-1 c$ to $G-1 b$ & Bølling to mid-Allerød & Allerød \\
\hline $14.1-13.9$ & GI-1d & Oldest Dryas & Older Dryas \\
\hline $14.67-14.1$ & GI-1e & Meiendorf & Bølling \\
\hline $16.5-14.67$ & GS-2 & & Oldest Dryas \\
\hline \multicolumn{4}{|l|}{$17.5-16.5$} \\
\hline \multicolumn{4}{|l|}{$19-17.5$} \\
\hline 20-19 & & & \\
\hline
\end{tabular}

\section{Results: geographic and chronological site distribution}

\subsection{0-19 ka cal BP - onset of deglaciation (31 dates, 20 sites)}

The earliest dates, at the onset of deglaciation between 20 and 19 ka cal BP, are Protomagdalenian and Badegoulian sites concentrated in southwest and central France, none further than $47^{\circ} \mathrm{N}$ (Fig. 2a). However, three AMS dates on reindeer bones, two with cut marks, ca. 23000 cal BP at Kastelhöhle Nord (Switzerland) and one date on a reindeer bone with cut marks from Y-Höhle cave indicate human settlement prior to the Magdalenian, attributed to the Badegoulian (see Leesch et al., 2012; Sedlmeier, 2010). The only dates at sites further north have been excluded: Hohle Fels (too old for the dated context, Street and Terberger, 1999) and Poggenwisch D (Hamburgian, improperly treated sediment samples, Weber and Grimm, 2009) in Germany.

\subsection{9-17.5 ka cal BP - cool, gradual warming phase (64 dates, 49 sites)}

During this gradual warming phase, the concentration of Lateglacial sites in southwest and central France persists. Initial expansion located above $47^{\circ} \mathrm{N}$ following a southwest-northeast axis is evidenced by the sites of Munzingen, Hohle Fels Schelklingen, Spitzbubenhöhle and Regensburg in southern Germany and as far east as Maszycka Cave in southern Poland (see Terberger et al., 2009 for discussion; see also Street et al., 2012; Kozłowski et al., 2012) (Fig. 2b).

The pattern during this phase shows an increase in population density in southwest and central France with some expansion northward. The northernmost sites in France are Oisy and Grotte du Renne à Arcy-sur-Cure, in proximity at $47.5^{\circ} \mathrm{N}$. Together, these southern German sites (Terberger et al., 2009; Jochim et al., 1999) and more northern French sites indicate early and probably nonintensive expansion northeast from southwest France.

Excluded dates for sites above $47^{\circ} \mathrm{N}$ include Rozel, Normandy $(17,000 \pm 700$ BP, Gif-1604; conventional date from 1970 (Delibrias et al., 1982), Hallines, Pas-de-Calais, (16,000 \pm 300 BP, Gif-1712, conventional date obtained in 1988 (Fagnard, 1988). The early dates from Trou des Blaireaux in Belgium have been rejected by Charles $(1996,1998)$ given lack of association with archaeological material or evidence of human modification. Finally, Kakert in Luxembourg yielded two dates $(16,070 \pm 450 \mathrm{BP}$, Lv-466; $16,770 \pm 390, \mathrm{Lv}-467$ ) which may be rejected given lack of association of the fauna with Magdalenian artifacts (Ziesaire, 1994). Two excluded sites not shown have been excluded given incompatibility between the dates and the archaeological context: Breitenbach B $(18,100 \pm 200 \mathrm{BP}, \mathrm{KN}-3332$, too young for the Aurignacian context, see Terberger and Street, 2003), and Poggenwisch D in northern Germany (Weber and Grimm, 2009).

\section{3. $17.5-16.5 \mathrm{ka} \mathrm{cal} \mathrm{BP}-$ Heinrich 1 event (or Oldest Dryas) (68 dates, 55 sites)}

This cold phase corresponds to the Heinrich 1 event, centered on 17 ka cal BP (Fig. 1). Three dates were obtained at the site of Gönnersdorf during this phase (13,810 \pm 90 BP, OxA-10200; $14,380 \pm 100$ BP, OxA-10239, $14570 \pm 90$ BP, OxA-10199). However, corresponding to a rhinoceros bone, a mammoth femur and mammoth ivory, all unworked, these dates do not appear to be associated with the Magdalenian occupation, but rather have been interpreted as sub-fossil material collected from the landscape and therefore much older than the Magdalenian occupation (Street and Terberger, 2004; Stevens et al., 2009: 133). In southern Germany, dates from Munzingen $(14,270 \pm 120$ BP, OxA-4788; 

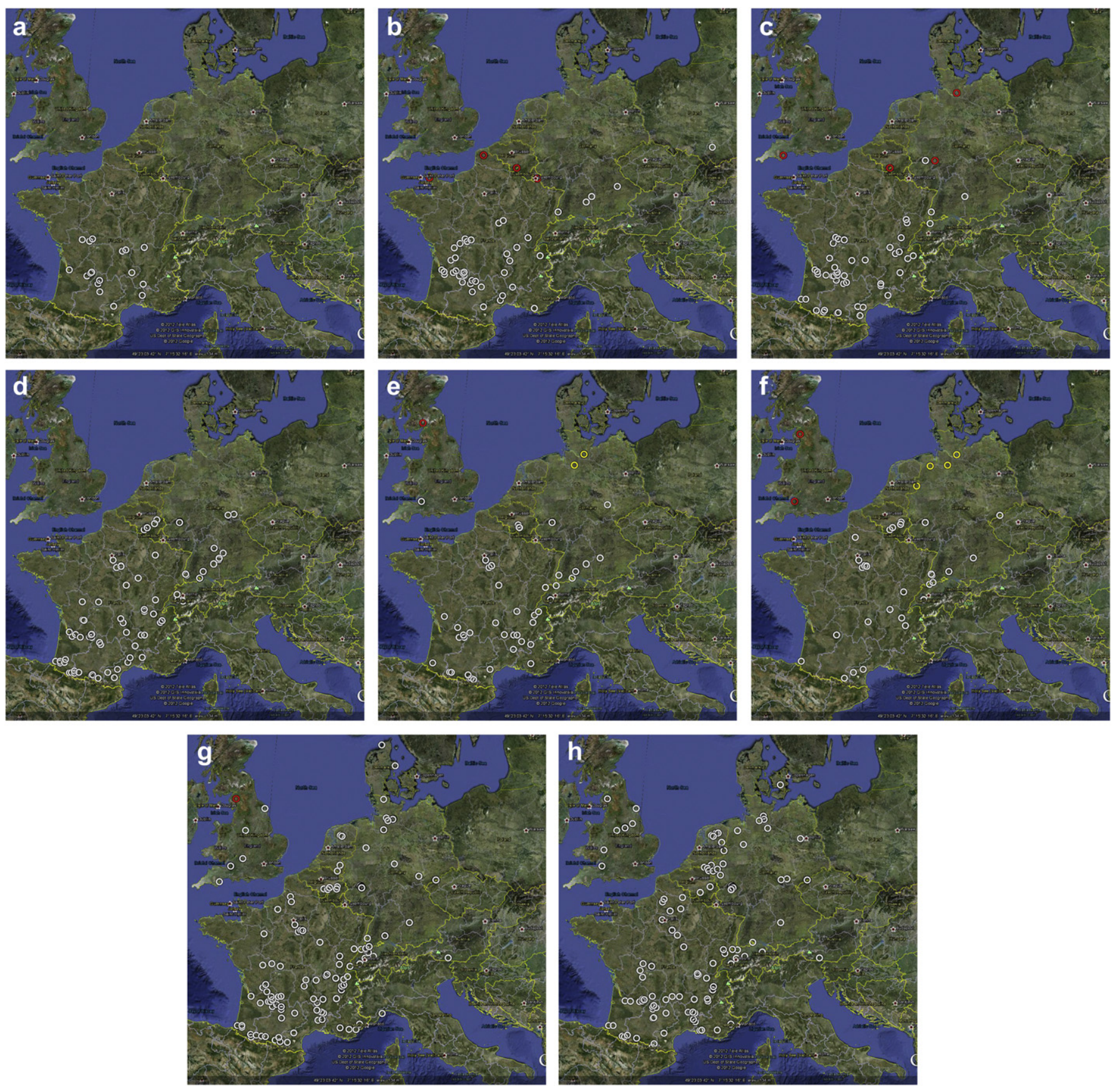

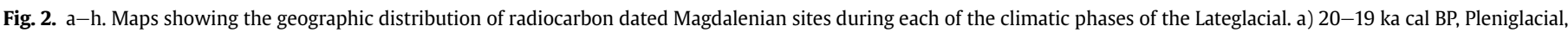

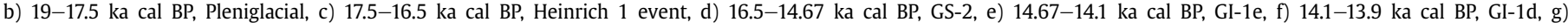
13.9-12.9 ka cal BP, GI-1c and 1b, h) 12.9-11.5 ka cal BP, GI-1a, GS-1. (Maps: Google Earth; site coordinates from the Radiocarbon Palaeolithic Database, version 12).

$14,510 \pm 110$ BP, OxA-4784) and Spitzbubenhöhle (13,840 $\pm 120 \mathrm{BP}$, H-4314-3715) may show persistence of human occupation during H1. However, the date for Spitzbubenhöhle is an old conventional radiocarbon date and could be excluded. Two sites are now found in Switzerland (Kesslerloch, 14,150 \pm 100 BP, OxA-5749; Kastelhöhle, $13,435 \pm 50$ BP, see; Leesch et al., 2012), following the same general southwest-northeast trajectory. The dates for Munzingen range from 12 to $16 \mathrm{ka}$ (see Housley et al., 1997 for discussion) and may be too broad, although comparisons have been made with Maszycka Cave in Poland (Kozłowski et al., 2012). Of these sites then, only dates from Kesslerloch, on worked reindeer antler, and Kastelhöhle on Bos/Bison, may be considered reliable. Northern France appears to have been as yet unoccupied while central and southwest France continue to show the same pattern as in the preceding phases. In brief, this phase is comparable to the preceding one, showing initial expansion from southwest to northeast via southern Germany and Switzerland (Fig. 2c).

Excluded dates for sites above $47^{\circ} \mathrm{N}$ include Kent's Cavern in England (14,275 $\pm 120 \mathrm{BP}, \mathrm{GrN}-6203$, questionable association with a Creswellian assemblage), Meiendorf D in Germany $(15,750 \pm 800 \mathrm{BP}, \mathrm{W}-172$, contaminated sediment sample, see Weber and Grimm, 2009), Trou des Blaireaux in Belgium $(13,790 \pm 150 \mathrm{BP}, \mathrm{Lv}-1314,13,930 \pm 120 \mathrm{BP}, \mathrm{Lv}-1433$, lack of association with artifacts or evidence of human modification, Charles, 
Table 2

Dates for 20-19 ka cal BP. (Note: All dates are conventional except for those indicated as AMS.)

\begin{tabular}{|c|c|c|c|c|c|c|c|}
\hline Site name & Country & Lab ID & Date & Uncertainty & From cal BP & To cal BP & Type \\
\hline Abri Fritsch & FR & Ly-1121 & 17,130 & 550 & 22,020 & 19,385 & \\
\hline Abri Fritsch & FR & Ly-1123 & 17,280 & 350 & 21,466 & 19,594 & \\
\hline Abri Pataud & FR & Grn-2064 & 17,605 & 420 & 22,170 & 20,012 & \\
\hline Abri Pataud & FR & GrN-5455 & 17,605 & 420 & 22,170 & 20,012 & \\
\hline Bouzil & FR & Ly-5113 & 18,715 & 1480 & 27,486 & 19,402 & \\
\hline Abri Casserole & FR & & 16,960 & 60 & 20,365 & 19,880 & \\
\hline Abri Casserole & FR & & 17,120 & 60 & 20,891 & 20088 & \\
\hline Champréveyres & $\mathrm{CH}$ & UCLA-2760 & 17,695 & 210 & 21,551 & 20,403 & AMS \\
\hline Gandil & FR & Ly-2485 (Poz) & 16,507 & 144 & 20,096 & 19,410 & \\
\hline La Contrée Viallet & FR & ARC-94 & 16,553 & 198 & 20,238 & 19,403 & \\
\hline Lascaux & FR & GrN-1632 & 17,190 & 140 & 21,132 & 20,061 & \\
\hline Lassac & FR & Gif-2981 & 16,750 & 250 & 20,459 & 19,419 & \\
\hline Laugerie-Haute Est & FR & Ly-973 & 17,040 & 440 & 21,426 & 19,425 & \\
\hline Le Cuzoul & FR & Gif-6312 & 16,800 & 170 & 20,347 & 19,543 & \\
\hline Le Cuzoul & FR & Gif-6371 & 16,800 & 170 & 20,347 & 19,543 & \\
\hline Le Cuzoul & FR & Gif-6797 & 17,050 & 170 & 20,990 & 19,590 & \\
\hline Le Roc de Marcamps & FR & Ly-2292 & 17,410 & 310 & 21,510 & 19,962 & \\
\hline Le Taillis-du-Coteau & FR & Ly-2264 & 16,920 & 170 & 20,467 & 19,565 & \\
\hline Les Guinards & FR & Ly-2737 & 17,420 & 330 & 21,549 & 19,885 & \\
\hline Les Jamblancs & FR & Gif-8668 & 16,490 & 130 & 20,050 & 19,411 & \\
\hline Monthaud & FR & Ly-2759 & 16,970 & 300 & 21,082 & 19,470 & \\
\hline Pégourié & FR & Ly-5257 & 16,890 & 300 & 21,019 & 19,436 & \\
\hline Pégourié & FR & Ly-1834 & 17,400 & 460 & 21,963 & 19,579 & \\
\hline Pégourié & FR & Ly-1836 & 17,420 & 390 & 21,776 & 19,596 & \\
\hline Pégourié & FR & Ly-1394 & 17,490 & 520 & 22,188 & 19,581 & \\
\hline Rond du Barry & FR & Gif-3038 & 17,100 & 450 & 21,480 & 19,431 & \\
\hline Salpêtrière & FR & Ly-940 & 17,900 & 690 & 23,273 & 19,611 & \\
\hline Salpêtrière & FR & Ly-941 & 17,900 & 690 & 23,273 & 19,611 & \\
\hline Salpêtrière & FR & Mc-2169 & 17,960 & 600 & 23,243 & 20,046 & \\
\hline Solutré, I-11 & FR & Ly-1534 & 17,310 & 470 & 21,805 & 19,474 & \\
\hline Solutré, L-13 & FR & Ly-316 & 17,350 & 300 & 21,455 & 19,926 & \\
\hline
\end{tabular}

$1996,1998)$ and Wildscheuer $(13,980 \pm 120 \mathrm{BP}, \mathrm{KN}-3637$, too young for Aurignacian context).

\subsection{5-14.67 ka cal BP - GS-2 (156 dates, 84 sites)}

This phase includes a fairly rapid increase in temperature from 16.5 to $14.67 \mathrm{ka}$ cal BP (although not observed in Switzerland, see Leesch et al., 2012), with an abrupt increase at $14.67 \mathrm{ka}$ cal BP, marking the pre-Bølling-Allerød (BA) to BA boundary (Liu et al., 2009, Fig. 1) between Greenland Stadial GS-2 and GI-1e (Litt et al. 2001, Table 3, p. 1247). Increasing climatic amelioration may have been a factor permitting expansion and/or seasonal occupation in more northern latitudes on a small scale compared with the denser settlement pattern subsequently observed during the Bølling (Fig. 2d). Champréveyres and Monruz in Switzerland have yielded a series of AMS dates that situate their occupations during this phase over into the next phase (see Leesch et al., 2012). Three dates have been excluded, Stafford in England (old conventional date), one date from Gönnersdorf $(12,910 \pm 105 \mathrm{BP}, \mathrm{KN}-1980$ on mollusk shell) and one from Andernach $(13,500 \pm 90 \mathrm{BP}$, OxA-13500, experimental sample; see Stevens et al., 2009).

Three key changes occur during this phase. First, the region of initial expansion into southern Germany is now more densely occupied by a series of sites (persistence at Munzingen and Spitzbubenhöhle, as well as new occupations at Petersfels, Hohle Fels, Bockstein-Törle, Hohlenstein-Kleine Scheuer, Hohlensteinstadel and Ofnet). Kesslerloch in northern Switzerland also falls within this region. Second, initial occupations of key sites in the Paris Basin occur during this phase (Etiolles, 13,625 \pm 105 BP, LYON-1894; Verberie, 12,950 \pm 130 BP, GifA-95454; and Grand Canton, $12,880 \pm 115$ BP, Gif-9608). Third, two site clusters are now present in Belgium, one in the upper Meuse basin near Dinant (Chaleux,
$12,990 \pm 140$ BP, Lv-1569; 13,000 \pm 200 BP, LC-919; Trou Da Somme, $12,815 \pm 75$ BP, OxA-8308; Trou des Blaireaux, $13,730 \pm 400 \mathrm{BP}, \mathrm{Lv}-1434 \mathrm{D} ; 13,850 \pm 335 \mathrm{BP}, \mathrm{Lv}-1309 \mathrm{D})$ and the other in eastern Belgium (Sy Verlaine, 13,780 \pm 220 BP, Lv-690; Grotte Walou, 13,030 \pm 140 BP, Lv-1582; 13,120 \pm 190 BP, Lv1593). These Belgian sites are at roughly the same latitude as Gönnersdorf, as are Kniegrotte $(13,585 \pm 165$, Bln-1564) and Baerenkeller (13,700 $\pm 380 \mathrm{BP}$, Bln-220) further east. The general pattern appears to be one of more intensive expansion, further north, with more substantial sites in the Paris Basin and the northern Rhineland.

\subsection{67-14.1 ka cal BP - GI-1e (Meiendorf [Litt et al., 2001] or traditional Bølling) (87 dates, 50 sites)}

This phase, corresponding to the stabilization of a warm phase, comprises relatively fewer radiocarbon dates for sites, but these show the same pattern as during the previous phase: a south-central France concentration with a path of expansion into southern Germany and a few sites north of $50^{\circ} \mathrm{N}$, but no longer with Magdalenian occupation in Switzerland (Leesch et al., 2012) (Fig. 2e). The range for the earliest date at Pincevent, one of the key Magdalenian sites in this region, corresponds to the end of the preceding phase/start of this phase $(12,545 \pm 120$ BP, GrN-5760, Taborin, 1994). Orp, in Belgium, has been dated by TL to this phase (Vermeersch and Maes, 1996). The appearance of the Hamburgian is represented by two sites in northern Germany, Poggenwisch D and Stellmoor D. The site of Roberthill in Scotland, dated in the early 1960s, has been excluded (Godwin, 1960; Moar, 1964). Sun Hole is the earliest dated Magdalenian site in Great Britain, based on recently obtained AMS dates on wild horse and a human ulna (Jacobi and Higham, 2011). 
Table 3

Dates for $19-17.5 \mathrm{ka}$ cal BP.

\begin{tabular}{|c|c|c|c|c|c|c|c|}
\hline Site name & Country & Lab ID & Date & Uncertainty & From cal BP & To cal BP & Type \\
\hline Abri de Gandil & FR & Gif-9175 & 15,550 & 140 & 19,241 & 18,520 & \\
\hline Abri Fritsch & FR & Ly-1122 & 16,530 & 550 & 21,195 & 18,714 & \\
\hline Abri Pataud & FR & GrN-2054 & 15,080 & 100 & 18,588 & 18,011 & \\
\hline Abri Pataud & FR & GrN-2254 & 15,215 & 250 & 18,898 & 17,858 & \\
\hline Auzary-Thones & FR & & 15,100 & 70 & 18,590 & 18,025 & \\
\hline Baume d'Oullins (a.k.a. d'Oulen") & FR & Gif-6017 & 16,500 & 350 & 20,434 & 18,872 & \\
\hline Baume du Lion & FR & MC-1209 & 16,200 & 400 & 20,252 & 18,657 & \\
\hline Baume du Lion & FR & MC- 1210 & 16,200 & 400 & 20,252 & 18,657 & \\
\hline Bergerie & FR & Ly-8692 & 15,320 & 110 & 18,825 & 18,083 & \\
\hline Bergerie & FR & Ly-1830 & 15,830 & 400 & 19,926 & 18,075 & \\
\hline Bois des Brousses & FR & Mc-2247 & 15,800 & 300 & 19,562 & 18,524 & \\
\hline Bois-des-Brousses & FR & MC-2247 & 15,800 & 300 & 19,562 & 18,524 & \\
\hline Chabasse & FR & Ly-1293 & 15,630 & 120 & 19,265 & 18,574 & \\
\hline Château & FR & Ly-10797 & 15,350 & 80 & 18,829 & 18,216 & \\
\hline Château & FR & Ly-10796 & 15,580 & 100 & 18,937 & 18,575 & \\
\hline Ferrassie & FR & Gif-2428 & 15,180 & 130 & 18,671 & 18,022 & \\
\hline Flageolet II & FR & Ly-918 & 15,250 & 320 & 19,263 & 17,714 & \\
\hline Gandil & FR & Ly-2483 (Poz) & 15,033 & 120 & 18,590 & 17,961 & \\
\hline Gandil & FR & Gif-9176 & 15,380 & 140 & 18,889 & 18,100 & \\
\hline Gazel & FR & Gif-2655 & 15,070 & 270 & 18,807 & 17,671 & \\
\hline Grappin & FR & Ly-457 & 15,320 & 370 & 19,394 & 17,787 & \\
\hline Grappin & FR & Ly-497 & 15,320 & 370 & 19,394 & 17,787 & \\
\hline Grappin & FR & Ly-559 & 15,770 & 390 & 19,862 & 18,050 & \\
\hline Grotte de la Bergerie & FR & Ly-8692 & 15,320 & 110 & 18,825 & 18,083 & \\
\hline Grotte du Renne, Arcy-sur-Cure & FR & L-340D & 15,350 & 400 & 19,416 & 17,737 & \\
\hline Hallines, Levert & FR & Gif-1712 & 16,000 & 300 & 19,846 & 18,619 & \\
\hline Hohle Fels, Hohler Fels & $\mathrm{DE}$ & H-5313-4898 & 15,760 & 140 & 19,358 & 18,664 & \\
\hline Kakert & LU & Lv-467 & 16,770 & 390 & 21,193 & 18,995 & \\
\hline La Chaire à Calvin & FR & Ly-1998 & 15,440 & 440 & 19,537 & 17,680 & \\
\hline La Garenne Grand Abri & FR & Ly-1126 & 15,560 & 580 & 20,146 & 17,571 & \\
\hline Lascaux & FR & SA-102 & 16,100 & 500 & 20,472 & 18,130 & \\
\hline Le Callan & FR & Gif-9663 & 15,020 & 70 & 18,550 & 18,005 & \\
\hline Le Chaffaud & FR & Gif- & 15,160 & 150 & 18,681 & 17,995 & \\
\hline Le Cuzoul & FR & Gif-6638 & 15,980 & 150 & 19,429 & 18,840 & \\
\hline Le Placard & FR & Gif-8803 & 16,300 & 190 & 19,930 & 18,897 & \\
\hline Le Roc de Marcamps & FR & Ly-2681 & 15,700 & 450 & 19,894 & 17,966 & \\
\hline Les Fadets & FR & & 15,300 & 150 & 18,841 & 18,038 & \\
\hline Les Jamblancs & FR & Gif-8667 & 14,850 & 130 & 18,529 & 17,712 & \\
\hline Les Terriers & FR & & 15,650 & 150 & 19,284 & 18,585 & \\
\hline Lespaux & FR & Ly-3307 & 17,450 & 780 & 22,977 & 18,991 & \\
\hline Maszycka & PL & Ly-2454 & 15,490 & 310 & 19,398 & 18,033 & \\
\hline Monthaud & FR & Ly-2758 & 15,450 & 290 & 19,334 & 18,025 & \\
\hline Munzingen & $\mathrm{DE}$ & OxA-4783 & 15,400 & 130 & 18,896 & 18,160 & AMS \\
\hline Munzingen & $\mathrm{DE}$ & OxA-4786 & 15,670 & 140 & 19,286 & 18,601 & AMS \\
\hline Munzingen & $\mathrm{DE}$ & ETH-7499 & 15,700 & 135 & 19,304 & 18,620 & AMS \\
\hline Munzingen & $\mathrm{DE}$ & $\mathrm{H}-4156-3373$ & 15,870 & 135 & 19,406 & 18,779 & \\
\hline Munzingen & $\mathrm{DE}$ & OxA-4785 & 16,060 & 140 & 19,445 & 18,873 & AMS \\
\hline Oetrange, Plateau Haed & LU & Lv-466 & 16,070 & 450 & 20,315 & 18,526 & \\
\hline Oisy & FR & Beta-160915 & 16,020 & 150 & 19,438 & 18,854 & \\
\hline Pégourié & FR & Ly-5258 & 16,090 & 320 & 19,990 & 18,651 & \\
\hline Pille-Bourse, Saint-Germain-la-Rivière & FR & Gif-5479 & 16,200 & 200 & 19,856 & 18,855 & \\
\hline Pille-Bourse, Saint-Germain-la-Rivière & FR & Gif-5478 & 15,300 & 410 & 19,407 & 17,667 & \\
\hline Regensburg, Florian-Seidl-Strasse & $\mathrm{DE}$ & $\mathrm{KN}-4264$ & 15,000 & 0 & 18,529 & 18,015 & \\
\hline Regensburg, Florian-Seidl-Strasse & $\mathrm{DE}$ & $\mathrm{KN}-4265$ & 15,000 & 0 & 18,529 & 18,015 & \\
\hline Roc de Marcamps & FR & Ly-2291 & 14,910 & 240 & 18,648 & 17,570 & \\
\hline Roc de Marcamps & FR & Ly-4222 & 15,070 & 270 & 18,807 & 17,671 & \\
\hline Roc De Marcamps & FR & Ly-4219 & 16,840 & 520 & 21,385 & 18,950 & \\
\hline Rond du Barry & FR & Gif-2672 & 15,400 & 400 & 19,433 & 17,777 & \\
\hline Rozel & FR & Gif-1604 & 17,000 & 700 & 22,102 & 18,878 & \\
\hline Sainte Eulalie & FR & Gif-1745 & 15,100 & 270 & 18,830 & 17,698 & \\
\hline Sainte Eulalie & FR & Gif-2194 & 15,200 & 300 & 19,195 & 17,652 & \\
\hline Schussenquelle & $\mathrm{DE}$ & H-860-970 & 15,900 & 360 & 19,926 & 18,510 & \\
\hline Solutré, i-11 & FR & Ly-9000 & 15,780 & 90 & 19,320 & 18,696 & \\
\hline Solutré, L-13 & FR & Ly-314 & 16,440 & 300 & 20,270 & 18,921 & \\
\hline Spitzbubenhöhle & $\mathrm{DE}$ & $\mathrm{H}-4149-3348$ & 15,230 & 100 & 18,699 & 18,036 & \\
\hline Trou des Blaireaux & $\mathrm{BE}$ & Lv-1558 & 16,130 & 250 & 19,876 & 18,759 & \\
\hline Trou des Blaireaux & $\mathrm{BE}$ & Lv-1385 & 16,270 & 230 & 20,002 & 18,872 & \\
\hline Vieux Mounoi & FR & Ly-5598 & 18,410 & 1680 & 27,635 & 18,806 & \\
\hline Vigne Brun & FR & Ly-2152 & 16,180 & 250 & 19,920 & 18,797 & \\
\hline
\end{tabular}


Table 4

Dates for 17.5-16.5 ka cal BP.

\begin{tabular}{|c|c|c|c|c|c|c|c|}
\hline Site name & Country & Lab ID & Date & Uncertainty & From cal BP & To cal BP & Type \\
\hline Abri Fritsch & FR & Ly-1001 & 14,960 & 380 & 18,874 & 17,206 & \\
\hline Auransan inf & FR & Ly-1107 & 13,910 & 230 & 17,655 & 16,567 & \\
\hline Auzary-Thones & FR & & 13,950 & 70 & 17,379 & 16,782 & \\
\hline Baume Noire, abri sud & FR & Ly-4716 & 13,950 & 135 & 17,455 & 16,775 & \\
\hline Baume Noire, abri sud & FR & Ly-4715 & 14,100 & 180 & 17,669 & 16,811 & \\
\hline Canecaude II & FR & Gif-2708 & 14,230 & 160 & 17,800 & 16,915 & \\
\hline Colombier & FR & Ly-5292 & 14,480 & 360 & 18,516 & 16,931 & \\
\hline Colombier & FR & Ly-5291 & 14,660 & 660 & 19,424 & 16,536 & \\
\hline Combe Cullier & FR & Ly-978 & 15,030 & 330 & 18,895 & 17,452 & \\
\hline Combe Saunière & FR & Ly-3328 & 13,910 & 230 & 17,655 & 16,567 & \\
\hline Conques & FR & Ly-8779 & 14,320 & 90 & 17,807 & 17,058 & \\
\hline Coufin & FR & Ly-2929 & 14,210 & 240 & 17,900 & 16,842 & \\
\hline Croze-sur-Suran, Grotte du Bare & FR & Ly-357 & 14,330 & 260 & 18,398 & 16,848 & \\
\hline Croze-sur-Suran, Grotte du Bare & FR & Ly-434 & 14,850 & 350 & 18,725 & 17,180 & \\
\hline Dufaure & FR & Ly-3582 & 14,570 & 390 & 18,577 & 16,943 & \\
\hline Duruthy & FR & Ly-860 & 13,840 & 210 & 17,554 & 16,505 & \\
\hline Duruthy & FR & Ly-861 & 14,180 & 200 & 17,808 & 16,855 & \\
\hline Enlène & FR & Gif-6030 & 13,900 & 120 & 17,409 & 16,742 & \\
\hline Enlène & FR & Gif-4124 & 13,940 & 250 & 17,756 & 16,566 & \\
\hline Esclauzure & FR & Ly-361 & 14,540 & 300 & 18,505 & 17,035 & \\
\hline Flageolet II & FR & Ly-1182 & 14,250 & 400 & 18,508 & 16,711 & \\
\hline Gönnersdorf & $\mathrm{DE}$ & OxA-10200 & 13,810 & 90 & 17,144 & 16,720 & AMS \\
\hline Gönnersdorf & $\mathrm{DE}$ & OxA-10239 & 14,380 & 100 & 17,875 & 17,128 & AMS \\
\hline Gönnersdorf & $\mathrm{DE}$ & OxA-10199 & 14,570 & 90 & 17,979 & 17,075 & AMS \\
\hline Grappin & FR & Ly-1535 & 14,530 & 290 & 18,504 & 17,019 & \\
\hline Grappin & FR & Ly-1510 & 14,820 & 370 & 18,740 & 17,132 & \\
\hline Grappin & FR & Ly-1536 & 14,840 & 360 & 18,738 & 17,162 & \\
\hline Grotte d'Aurensan, Diogène & FR & Ly-1055 & 14,280 & 300 & 18,435 & 16,803 & \\
\hline Igue du Gral & FR & Ly-11518 & 13,682 & 74 & 17,019 & 16,618 & \\
\hline Igue du Gral & FR & Ly-12418 & 13,970 & 90 & 17,417 & 16,795 & \\
\hline Jaurias & FR & Gd-2697 & 14,660 & 200 & 18,506 & 17,230 & \\
\hline Kastelhöhle & $\mathrm{CH}$ & B-4636 & 13,900 & 150 & 17,448 & 16,730 & \\
\hline Kent's Cavern & UK & GrN-6203 & 14,275 & 120 & 17,787 & 16,980 & \\
\hline La Colombière & FR & Ly-?1 & 14,700 & 300 & 18,575 & 17,165 & \\
\hline La Colombière & FR & Ly-?2 & 15,500 & 700 & 20,302 & 17,171 & \\
\hline La Garenne Grand Abri & FR & Ly-3000 & 14,270 & 270 & 18,015 & 16,825 & \\
\hline La Garenne Grand abri & FR & Ly-1125 & 15,330 & 950 & 21,199 & 16,739 & \\
\hline La Garenne-Blanchard & FR & C-578 & 15,847 & 1220 & 22,660 & 16,716 & \\
\hline La Marche & FR & Ly-2100 & 14,280 & 160 & 17,850 & 16,960 & \\
\hline La Marche, Réseau Guy Martin & FR & Orsay-3780 & 14,240 & 85 & 17,663 & 16,971 & \\
\hline Labastide, La Grande Grotte & FR & Gif-6611 & 13,700 & 120 & 17,105 & 16,531 & \\
\hline Labastide, La Grande Grotte & FR & Ly-1405 & 14,260 & 440 & 18,568 & 16,589 & \\
\hline Lascaux & FR & C-406 & 15,516 & 900 & 21,080 & 16,921 & \\
\hline Laugerie-Basse & FR & Gif-5387 & 13,850 & 160 & 17,435 & 16,663 & \\
\hline Le Bay & FR & & 13,950 & 50 & 17,221 & 16,790 & \\
\hline Le Cuzoul & FR & Gif-6372 & 14,560 & 130 & 18,380 & 17,185 & \\
\hline Le Martinet & FR & Ly-5069 & 14,098 & 239 & 17,822 & 16,785 & \\
\hline Le Taillis-du-Coteau & FR & Ly-3876 & 14,630 & 75 & 18,382 & 17,445 & \\
\hline Les Cottes [St. Pierre de Maille] & FR & GrN-4457 & 14,360 & 150 & 17,903 & 17,033 & \\
\hline Les Jamblancs & FR & Gif-8666 & 13,790 & 120 & 17,184 & 16,654 & \\
\hline Les Jamblancs & FR & Gif-8669 & 13,900 & 110 & 17,395 & 16,744 & \\
\hline Meiendorf D & $\mathrm{DE}$ & W-172 & 15,750 & 800 & 21,063 & 17,246 & \\
\hline Monruz & $\mathrm{CH}$ & ETH-6413 & 13,330 & 110 & 16,829 & 15,570 & AMS \\
\hline Monruz & $\mathrm{CH}$ & ETH-6421 & 13,140 & 120 & 16,591 & 15,211 & AMS \\
\hline Monruz & $\mathrm{CH}$ & ETH-6420 & 13,120 & 120 & 16,564 & 15195 & AMS \\
\hline Monruz & $\mathrm{CH}$ & ETH-6418 & 13,110 & 120 & 16,550 & 15,188 & AMS \\
\hline Monruz & $\mathrm{CH}$ & ETH-6416 & 13,070 & 130 & 16,519 & 15,152 & AMS \\
\hline Montgaudier Abri Gaudry & FR & BM-2309 & 14,770 & 270 & 18,582 & 17248 & \\
\hline Moulin-Neuf & FR & Ly-2275 & 14,280 & 440 & 18,570 & 16,626 & \\
\hline Munzingen & DE & OxA-4788 & 14,270 & 120 & 17,780 & 16,975 & AMS \\
\hline Munzingen & $\mathrm{DE}$ & OxA-4784 & 14,510 & 110 & 17,974 & 17,210 & AMS \\
\hline Rigney & FR & Ly-1191 & 14,950 & 500 & 19,263 & 17,029 & \\
\hline Roc de Marcamps & FR & Ly-2290 & 14,200 & 190 & 17,812 & 16,874 & \\
\hline Roc-aux-Sorciers, Abri Bourdois & FR & GrN-1903 & 13,920 & 80 & 17,237 & 16,761 & \\
\hline Roc-aux-Sorciers, Abri Bourdois & FR & GrN-1913 & 14,160 & 80 & 17,571 & 16,923 & \\
\hline Romains & FR & Ly-16 & 14,380 & 380 & 18,503 & 16,841 & \\
\hline Saint-Germain-la-Rivière & FR & Gif-6037 & 14,100 & 160 & 17,627 & 16,829 & \\
\hline Salpêtrière & FR & MC-1368 & 14,200 & 300 & 18,038 & 16,749 & \\
\hline Schussenquelle & $\mathrm{DE}$ & GRO-468 & 14,470 & 385 & 18,530 & 16,896 & \\
\hline Solutré, I-11 & FR & Ly-1532 & 14,360 & 280 & 18,450 & 16,860 & \\
\hline Spitzbubenhöhle & $\mathrm{DE}$ & $\mathrm{H}-4314-3715$ & 13,840 & 120 & 17,233 & 16,677 & \\
\hline Tournal (or Grande Grotte de Bize) & FR & Ly-1232 & 14,530 & 510 & 18,803 & 16,754 & \\
\hline Trou des Blaireaux & $\mathrm{BE}$ & LV-1314 & 13,790 & 150 & 17,381 & 16,560 & \\
\hline Trou des Blaireaux & $\mathrm{BE}$ & Lv-1433 & 13,930 & 120 & 17,420 & 16,767 & \\
\hline
\end{tabular}


Table 4 (continued)

\begin{tabular}{|c|c|c|c|c|c|c|c|}
\hline Site name & Country & Lab ID & Date & Uncertainty & From cal BP & To cal BP & Type \\
\hline Tuc d'Audoubert & FR & Gif-5867 & 14,350 & 160 & 17,905 & 17,011 & \\
\hline Tuc d'Audoubert, Cheval Rouge & FR & Gif-5857 & 14,350 & 160 & 17,905 & 17,011 & \\
\hline Wildscheuer & $\mathrm{DE}$ & KN-3637 & 13,980 & 120 & 17,452 & 16,799 & \\
\hline
\end{tabular}

Table 5

Dates for $16.5-14.67 \mathrm{ka}$ cal BP.

\begin{tabular}{|c|c|c|c|c|c|c|c|}
\hline Site name & Country & Lab ID & Date & Uncertainty & From cal BP & To cal BP & Type \\
\hline Abri Ragot, Bois Ragot & FR & GrN-4677 & 12,890 & 140 & 16,401 & 14,926 & \\
\hline Andernach Martinsberg 1 & $\mathrm{DE}$ & OxA-18409 & 13,025 & 50 & 16,343 & 15,163 & AMS \\
\hline Andernach Martinsberg 1 & $\mathrm{DE}$ & OxA-10493 & 13,185 & 80 & 16,623 & 15,290 & AMS \\
\hline Andernach Martinsberg 1 & $\mathrm{DE}$ & OxA-10651 & 13,270 & 180 & 16,790 & 15,270 & AMS \\
\hline Andernach Martinsberg 1 & $\mathrm{DE}$ & OxA-10492 & 13,500 & 90 & 16,928 & 16,220 & AMS \\
\hline Andernach-Martinsberg & $\mathrm{DE}$ & OxA-1126 & 12,890 & 140 & 16,401 & 14,926 & AMS \\
\hline Andernach-Martinsberg & $\mathrm{DE}$ & OxA-1125 & 12,930 & 180 & 16,559 & 14,894 & AMS \\
\hline Andernach-Martinsberg & $\mathrm{DE}$ & OxA-V-2218-38 & 13,015 & 50 & 16,327 & 15,152 & AMS \\
\hline Andernach-Martinsberg & $\mathrm{DE}$ & OxA-1129 & 13,090 & 130 & 16,543 & 15,166 & AMS \\
\hline Andernach-Martinsberg & $\mathrm{DE}$ & OxA-V-2218-40 & 13,110 & 50 & 16,458 & 15,240 & AMS \\
\hline Andernach-Martinsberg & $\mathrm{DE}$ & GrA-16985 & 13,110 & 80 & 16,490 & 15,217 & AMS \\
\hline Andernach-Martinsberg & $\mathrm{DE}$ & OxA-V-2216-43 & 13,135 & 55 & 16,502 & 15,262 & AMS \\
\hline Andernach-Martinsberg & $\mathrm{DE}$ & GrA-16986 & 13,180 & 70 & 16,608 & 15,297 & AMS \\
\hline Andernach-Martinsberg & $\mathrm{DE}$ & OxA-1128 & 13,200 & 140 & 16,687 & 15,242 & AMS \\
\hline Baerenkeller & $\mathrm{DE}$ & Bln-220 & 13,700 & 380 & 17,668 & 15,251 & \\
\hline Baume de Gigny & FR & Ly-1702 & 13,620 & 480 & 17,896 & 15,060 & \\
\hline Baume du Lion & FR & MC-2448 & 13,100 & 300 & 16,868 & 14,811 & \\
\hline Belvis & FR & SacA-6973 & 13,080 & 50 & 16,417 & 15,214 & \\
\hline Blanzat & FR & & 12,870 & 70 & 16,055 & 14,967 & \\
\hline Blot & FR & Ly-563 & 14,030 & 500 & 18,519 & 15,630 & \\
\hline Bockstein-Torle & $\mathrm{DE}$ & $\mathrm{H}-4057-3354$ & 12,980 & 103 & 16,374 & 15,102 & \\
\hline Bois du Cantet & FR & Ly-1403 & 13,370 & 270 & 16,926 & 15,202 & \\
\hline Bois-des-Brousses & FR & Gif-6013 & 13,500 & 230 & 17,073 & 15,300 & \\
\hline Bourrouilla & FR & Gif-10255 & 12,780 & 40 & 15,591 & 14,910 & \\
\hline Bourrouilla & FR & Gif-10254 & 13,220 & 80 & 16,692 & 15,389 & \\
\hline Calvaire & FR & Ly-432 & 13,450 & 300 & 17,073 & 15,190 & \\
\hline Campalou & FR & Ly-1958 & 13,400 & 350 & 17,170 & 15,080 & \\
\hline Champréveyres & $\mathrm{CH}$ & UZ-2285 & 13,050 & 155 & 16,550 & 15,116 & AMS \\
\hline Champréveyres & $\mathrm{CH}$ & UZ-2283 & 12,950 & 155 & 16,474 & 15,004 & AMS \\
\hline Champréveyres & $\mathrm{CH}$ & UZ-2286 & 12,870 & 135 & 16,386 & 14,900 & AMS \\
\hline Champréveyres & $\mathrm{CH}$ & UZ-2282 & 12,825 & 155 & 16,359 & 14,605 & AMS \\
\hline Champréveyres & $\mathrm{CH}$ & OxA-20700 & 12,815 & 65 & 15,862 & 14,912 & AMS \\
\hline Champréveyres & $\mathrm{CH}$ & OxA-20701 & 12,805 & 75 & 15,884 & 14,889 & AMS \\
\hline Champréveyres & $\mathrm{CH}$ & UZ-2171 & 12,730 & 135 & 15,892 & 14,238 & AMS \\
\hline Champréveyres & $\mathrm{CH}$ & UZ-2175 & 12,630 & 130 & 15,493 & 14,166 & AMS \\
\hline Champréveyres & $\mathrm{CH}$ & UZ-2172 & 12,620 & 145 & 15,505 & 14,142 & AMS \\
\hline Champréveyres & $\mathrm{CH}$ & UZ-2177 & 12,600 & 145 & 15,479 & 14,122 & AMS \\
\hline Champréveyres & $\mathrm{CH}$ & UZ-2173 & 12,540 & 140 & 15,194 & 14,106 & AMS \\
\hline Champréveyres & $\mathrm{CH}$ & UZ-2174 & 12,510 & 130 & 15,147 & 14,121 & AMS \\
\hline Champréveyres & $\mathrm{CH}$ & UZ-2287 & 12,500 & 145 & 15,156 & 14,079 & AMS \\
\hline Comarque & FR & Ly-2154 & 13,370 & 340 & 17,104 & 15,083 & \\
\hline Combarelles & FR & Ly-3202 & 13,680 & 210 & 17,415 & 15,929 & \\
\hline Conques & FR & Ly-8778 & 13,330 & 140 & 16,850 & 15,476 & \\
\hline Dufaure & FR & Ly-3583 & 14,020 & 340 & 18,410 & 16,330 & \\
\hline Durif & FR & Ly-2046 & 13,090 & 270 & 16,819 & 14,951 & \\
\hline Durif & FR & Ly-3675 & 13,510 & 230 & 17,093 & 15,435 & \\
\hline Durif à Enval & FR & Ly-727 & 13,700 & 380 & 17,668 & 15,251 & \\
\hline Duruthy & FR & Ly-859 & 13,510 & 220 & 17,077 & 15,477 & \\
\hline Ebbou, Ebbe, Chateau d'Ebbo & FR & Ly-800 & 12,980 & 220 & 16,701 & 14,876 & \\
\hline Enlène & FR & Gif-5321 & 12,990 & 140 & 16,461 & 15,079 & \\
\hline Enlène & FR & Gif-5770 & 13,400 & 120 & 16,879 & 15,620 & \\
\hline Espalungue & FR & Ly-3481 & 12,970 & 160 & 16,499 & 15,021 & \\
\hline Espelugue & FR & Ly-1406 & 13,170 & 260 & 16,812 & 15,102 & \\
\hline Etiolles, Les Coudrays & FR & LYON-1894 & 13,625 & 105 & 17,010 & 16,456 & AMS \\
\hline Flageolet II & FR & Ly-917 & 14,110 & 690 & 18,788 & 15,166 & \\
\hline Fongaban & FR & Ly-977 & 14,300 & 680 & 18,932 & 15,274 & \\
\hline Gnirshohle 1 & $\mathrm{DE}$ & $\mathrm{H}-6273-5833$ & 12,830 & 100 & 16,137 & 14,875 & \\
\hline Gönnersdorf & $\mathrm{DE}$ & $\mathrm{KN}-1980$ & 12,910 & 105 & 16,290 & 15,009 & AMS \\
\hline Gönnersdorf & $\mathrm{DE}$ & OxA-5729 & 12,910 & 130 & 16,380 & 14,987 & AMS \\
\hline Gönnersdorf & $\mathrm{DE}$ & OxA-V-2223-42 & 12,990 & 55 & 16,282 & 15,120 & AMS \\
\hline Gönnersdorf & $\mathrm{DE}$ & OxA-V-2222-31 & 13,010 & 55 & 16,327 & 15,146 & AMS \\
\hline Gönnersdorf & $\mathrm{DE}$ & OxA-15295 & 13,060 & 60 & 16,402 & 15,192 & AMS \\
\hline Gönnersdorf & $\mathrm{DE}$ & OxA-V-2223-43 & 13,075 & 55 & 16,416 & 15,206 & AMS \\
\hline Gönnersdorf & $\mathrm{DE}$ & OxA-V-2223-41 & 13,095 & 55 & 16,441 & 15,223 & AMS \\
\hline Gönnersdorf & $\mathrm{DE}$ & OxA-V-2223-40 & 13,165 & 55 & 16,565 & 15,298 & AMS \\
\hline
\end{tabular}


Table 5 (continued)

\begin{tabular}{|c|c|c|c|c|c|c|c|}
\hline Site name & Country & Lab ID & Date & Uncertainty & From cal BP & To cal BP & Type \\
\hline Gönnersdorf & $\mathrm{DE}$ & OxA-V-2223-39 & 13,270 & 55 & 16,734 & 15,567 & AMS \\
\hline Gönnersdorf & $\mathrm{DE}$ & OxA-10201 & 13,610 & 80 & 16,973 & 16,499 & AMS \\
\hline Goutte Roffat & FR & Ly-3092 & 13,350 & 260 & 16,897 & 15,210 & \\
\hline Grand Canton & FR & Gif-9608 & 12,880 & 115 & 16,289 & 14,942 & \\
\hline Grande Galerie 2 & FR & & 13,900 & 600 & 18,513 & 15,176 & \\
\hline Grappin & FR & Ly-1509 & 14,220 & 560 & 18,704 & 15,656 & \\
\hline Grotte de Laroque II, Roque & FR & & 13,100 & 300 & 16,868 & 14,811 & \\
\hline Hohle Fels, Hohler Fels & $\mathrm{DE}$ & H-5119-4601 & 13,085 & 95 & 16,477 & 15,189 & \\
\hline Hohlenstein, Kleine Scheuer & $\mathrm{DE}$ & $\mathrm{H}-4183-3416$ & 13,252 & 98 & 16,765 & 15,430 & \\
\hline Hohlenstein-Stadel & $\mathrm{DE}$ & H-3799-3045 & 13,110 & 160 & 16,617 & 15,161 & \\
\hline Hohlenstein-Stadel & $\mathrm{DE}$ & H-3779-3044 & 13,550 & 130 & 17,007 & 16,092 & \\
\hline Igue du Gral & FR & Ly-12419 & 13,360 & 70 & 16,850 & 15,675 & \\
\hline Jaurias & FR & Gd-2698 & 13,500 & 200 & 17,028 & 15,539 & \\
\hline Jaurias & FR & Ly-3730 & 13,580 & 140 & 17,050 & 16,119 & \\
\hline Jean Pierre II & FR & Ly-830 & 13,070 & 210 & 16,685 & 15,074 & \\
\hline Jean Pierre II & FR & Ly-926 & 13,280 & 290 & 16,897 & 15,127 & \\
\hline Jean Pierre II & FR & Ly-390 & 13,300 & 280 & 16,891 & 15,156 & \\
\hline Kesslerloch & $\mathrm{CH}$ & OxA-5748 & 12,770 & 90 & 15,855 & 14,652 & AMS \\
\hline Kesslerloch & $\mathrm{CH}$ & Hv-10652 & 12,890 & 90 & 16,166 & 14,976 & \\
\hline Kesslerloch & $\mathrm{CH}$ & B-3329 & 12,970 & 180 & 16,561 & 14,979 & \\
\hline Kesslerloch & $\mathrm{CH}$ & OxA-5746 & 13,120 & 90 & 16,518 & 15,217 & AMS \\
\hline Kesslerloch & $\mathrm{CH}$ & OxA-5747 & 13,430 & 100 & 16,890 & 15,909 & AMS \\
\hline Kesslerloch & $\mathrm{CH}$ & OxA-5750 & 13,670 & 100 & 17,044 & 16,541 & AMS \\
\hline Kesslerloch & $\mathrm{CH}$ & OxA-5749 & 14,150 & 100 & 17,585 & 16,900 & AMS \\
\hline Kniegrotte & $\mathrm{DE}$ & Bln-1564 & 13,585 & 165 & 17,082 & 15,938 & \\
\hline La Colombière & FR & Ly-177 & 14,150 & 450 & 18,565 & 16,224 & \\
\hline La Colombière & FR & Ly-9713 & 14,390 & 700 & 19,267 & 15,600 & \\
\hline La Colombière & FR & Ly-433 & 13,390 & 300 & 17,000 & 15,164 & \\
\hline La Garenne Grand Abri & FR & Ly-1127 & 14,080 & 350 & 18,448 & 16,453 & \\
\hline La Madeleine & FR & Ly-921 & 13,070 & 190 & 16,641 & 15,100 & \\
\hline La Madeleine & FR & Ly-922 & 13,440 & 300 & 17,060 & 15,185 & \\
\hline Labastide, La Grande Grotte & FR & Gif-6612 & 13,500 & 120 & 16,941 & 15,976 & \\
\hline Lascaux & FR & GIN-4677 & 12,890 & 140 & 16,401 & 14,926 & \\
\hline Laugerie-Haute Est & FR & Ly-974 & 13,970 & 480 & 18,498 & 15,528 & \\
\hline Malarode & FR & Ly-3484 & 13,620 & 320 & 17,480 & 15,275 & \\
\hline Mas-d'Azil & FR & Gif-5680 & 13,200 & 100 & 16,660 & 15,283 & \\
\hline Mas-d'Azil & FR & Gif-5522 & 13,640 & 110 & 17,030 & 16,464 & \\
\hline Monruz & $\mathrm{CH}$ & OxA-20699 & 13,055 & 60 & 16,395 & 15,188 & AMS \\
\hline Monruz & $\mathrm{CH}$ & ETH-6417 & 13,030 & 120 & 16,456 & 15,131 & AMS \\
\hline Monruz & $\mathrm{CH}$ & ETH-6412 & 12,970 & 110 & 16,378 & 15,089 & AMS \\
\hline Monruz & $\mathrm{CH}$ & ETH-6415 & 12,900 & 120 & 16,338 & 14,982 & AMS \\
\hline Monruz & $\mathrm{CH}$ & ETH-6419 & 12,880 & 120 & 16,314 & 14,940 & AMS \\
\hline Monruz & $\mathrm{CH}$ & ETH-6414 & 12,840 & 120 & 16,301 & 14,801 & AMS \\
\hline Monruz & $\mathrm{CH}$ & ETH-20727 & 12,800 & 85 & 15,905 & 14,759 & AMS \\
\hline Montgaudier Abri Paignon & FR & BM-1916 & 13,320 & 360 & 17,137 & 14,969 & \\
\hline Monthaud & FR & Ly-2757 & 13,420 & 200 & 16,942 & 15,300 & \\
\hline Moulin-du-Roc & FR & Ly-5445 & 15,600 & 1200 & 22,446 & 16,429 & \\
\hline Moulin-Neuf & FR & Ly-2699 & 13,380 & 250 & 16,911 & 15,238 & \\
\hline Moulin-Neuf & FR & Ly-2352 & 13,570 & 260 & 17,207 & 15,288 & \\
\hline Munzingen & $\mathrm{DE}$ & OxA-4820 & 13,230 & 110 & 16,720 & 15,291 & AMS \\
\hline Munzingen & $\mathrm{DE}$ & ETH-7500 & 13,560 & 120 & 17,007 & 16,219 & AMS \\
\hline Ofnet & $\mathrm{DE}$ & UCLA-1783 & 13,100 & 100 & 16,505 & 15,195 & \\
\hline Oisy & FR & Beta-160914 & 13,260 & 80 & 16,757 & 15,501 & \\
\hline Pégourié & FR & Ly-1598 & 13,980 & 510 & 18,509 & 15,521 & \\
\hline Petersfels & $\mathrm{DE}$ & H-6656-6793 & 12900 & 90 & 16,192 & 14,993 & \\
\hline Petersfels & $\mathrm{DE}$ & H-5211-4891 & 12,940 & 125 & 16,388 & 15,040 & \\
\hline Petersfels & $\mathrm{DE}$ & H-7142-7348 & 12,980 & 90 & 16,347 & 15,108 & \\
\hline Petersfels & $\mathrm{DE}$ & H-7143-7301 & 13,030 & 100 & 16,422 & 15,146 & \\
\hline Petersfels & $\mathrm{DE}$ & H-7216-7363 & 13,110 & 90 & 16,504 & 15,210 & \\
\hline Peyrugues & FR & Gif-7592 & 13,020 & 140 & 16,486 & 15,105 & \\
\hline Roc de Marcamps & FR & Ly-2680 & 13,570 & 420 & 17,631 & 15,111 & \\
\hline Romains & FR & Ly-356 & 12,980 & 240 & 16,718 & 14,693 & \\
\hline Romains & FR & GrA9710 (Lyon-643) & 13,380 & 60 & 16,843 & 15,943 & AMS \\
\hline Saint-Mihiel & FR & Lv-2096 & 13,160 & 110 & 16,605 & 15,235 & \\
\hline Salpêtrière & FR & MC-920 & 13,100 & 200 & 16,680 & 15,116 & \\
\hline Schnurenloch & $\mathrm{CH}$ & B-157 & 14,000 & 600 & 18,546 & 15,258 & \\
\hline Schussenquelle & DE & $\mathrm{KN}-4250$ & 12,860 & 120 & 16,308 & 14,904 & \\
\hline Schussenquelle & $\mathrm{DE}$ & $\mathrm{KN}-4251$ & 13,050 & 120 & 16,477 & 15,146 & \\
\hline Schussenquelle & $\mathrm{DE}$ & GrN-2090 & 13,090 & 110 & 16,508 & 15,181 & \\
\hline Solutré, I-11 & FR & Ly-1530 & 13,680 & 240 & 17,482 & 15,655 & \\
\hline Solutré, I-11 & FR & Ly-1531 & 13,710 & 230 & 17,493 & 15,931 & \\
\hline Solutré, P16 & FR & Ly- & 13,350 & 350 & 17,125 & 15,040 & \\
\hline Spitzbubenhöhle & $\mathrm{DE}$ & $\mathrm{H}-4052-3212$ & 12,747 & 10 & 15,538 & 14,896 & \\
\hline Stafford & UK & BIRM-150 & 13,490 & 375 & 17,415 & 15,122 & \\
\hline Sy-Verlaine & $\mathrm{BE}$ & Lv-690 & 13,780 & 220 & 17,567 & 16,301 & \\
\hline
\end{tabular}


Table 5 (continued)

\begin{tabular}{|c|c|c|c|c|c|c|c|}
\hline Site name & Country & Lab ID & Date & Uncertainty & From cal BP & To cal BP & Type \\
\hline Teufelsbrücke & $\overline{\mathrm{DE}}$ & Bln-1573 & 13,025 & 85 & 16,392 & 15,151 & \\
\hline Tournal (or Grande Grotte de Bize) & FR & Ly-1897 & 13,790 & 420 & 17,909 & 15,271 & \\
\hline Tournal (or Grande Grotte de Bize) & FR & Ly-1675 & 14,770 & 970 & 20,308 & 15,260 & \\
\hline Trou Da Somme & $\mathrm{BE}$ & OxA-8308 & 12,815 & 75 & 15,893 & 14,901 & AMS \\
\hline Trou de Chaleux & $\mathrm{BE}$ & Lv-1569 & 12,990 & 140 & 16,461 & 15,079 & \\
\hline Trou de Chaleux & $\mathrm{BE}$ & MC-919 & 13,000 & 200 & 16,636 & 14,987 & \\
\hline Trou des Blaireaux & $\mathrm{BE}$ & Lv-1434D & 13,730 & 400 & 17769 & 15,260 & \\
\hline Trou des Blaireaux & $\mathrm{BE}$ & Lv-1309D & 13,850 & 335 & 17,903 & 15,666 & \\
\hline Trou du Frontal & $\mathrm{BE}$ & Lv-1749 & 12,950 & 170 & 16,519 & 14,964 & \\
\hline Trou du Frontal & $\mathrm{BE}$ & Lv-1750 & 13,130 & 170 & 16,651 & 15,168 & \\
\hline Trou Walou & $\mathrm{BE}$ & LV-1582 & 13,030 & 140 & 16,496 & 15,113 & \\
\hline Trou Walou & $\mathrm{BE}$ & Lv-1593 & 13,120 & 190 & 16,674 & 15,144 & \\
\hline Tuc d'Audoubert, Cheval Rouge & FR & KN-5091 & 13,340 & 120 & 16,840 & 15,560 & \\
\hline Tuc d'Audoubert, Cheval Rouge & FR & KN-5090 & 13,480 & 80 & 16,910 & 16,212 & \\
\hline Vache & FR & GrN-2026 & 12,850 & 60 & 15,889 & 14,964 & \\
\hline Verberie, Le Buisson Campin & FR & GifA-95454 & 12,950 & 130 & 16,409 & 15,046 & AMS \\
\hline Vidon & FR & Ly-2701 & 14,000 & 350 & 18,410 & 16,161 & \\
\hline
\end{tabular}

Table 6

Dates for 14.67-14.1 ka cal BP.

\begin{tabular}{|c|c|c|c|c|c|c|c|}
\hline Site name & Country & Lab ID & Date & Uncertainty & From cal BP & To cal BP & Type \\
\hline Abri Taillefer & FR & Ly-637 & 12,590 & 60 & 15,190 & 14,245 & \\
\hline Adaouste & FR & Ly-540 & 12,760 & 250 & 16,411 & 14,157 & \\
\hline Adaouste & FR & LGQ-127 & 12,981 & 266 & 16,740 & 14,626 & \\
\hline Andernach-Martinsberg & $\mathrm{DE}$ & OxA-V-2223.37 & 12,675 & 55 & 15,460 & 14,596 & AMS \\
\hline Andernach-Martinsberg & $\mathrm{DE}$ & OxA-1127 & 12,820 & 130 & 16,209 & 14,637 & AMS \\
\hline Baume d'Oullins & FR & Gif-6016 & 12,800 & 180 & 16,395 & 14,253 & \\
\hline Bois du Cantet & FR & Ly-1404 & 13,060 & 430 & 16,910 & 14,189 & \\
\hline Bois Laiterie & $\mathrm{BE}$ & GX-20433 & 12,625 & 117 & 15,453 & 14,174 & \\
\hline Bois Laiterie & $\mathrm{BE}$ & GX-20434 & 12,665 & 96 & 15,520 & 14,244 & \\
\hline Bourrouilla & FR & Gif-10234 & 12,710 & 90 & 15,604 & 14,553 & \\
\hline Bourrouilla & FR & Gif-10235 & 12,710 & 90 & 15,604 & 14,553 & \\
\hline Bourrouilla, Grotte d'Arancou & FR & Gif-9986 & 12,395 & 35 & 14,921 & 14,113 & \\
\hline Calvaire & FR & Ly-431 & 12,970 & 300 & 16,781 & 14,270 & \\
\hline Campalou & FR & Ly-436 & 12,800 & 300 & 16,555 & 14,141 & \\
\hline Comarque & FR & Ly-2355 & 12,710 & 200 & 16,150 & 14,135 & \\
\hline Crest & FR & Ly-894 & 12,850 & 240 & 16,539 & 14,241 & \\
\hline Douattes & FR & Ly-647 & 12,680 & 60 & 15,480 & 14,595 & AMS \\
\hline Durif à Enval & FR & Ly-425 & 13,000 & 300 & 16,804 & 14,556 & \\
\hline Eglises & FR & Gif-3923 & 12,900 & 220 & 16,600 & 14,577 & \\
\hline Etiolles, Les Coudrays & FR & OxA-173 & 12,800 & 220 & 16,400 & 14,238 & AMS \\
\hline Etiolles, Les Coudrays & FR & OxA-175 & 12,900 & 220 & 16,600 & 14,577 & AMS \\
\hline Etiolles, Les Coudrays & FR & OxA-138 & 12,990 & 300 & 16,802 & 14,536 & AMS \\
\hline Etiolles, Les Coudrays & FR & OxA-139 & 13,000 & 300 & 16,804 & 14,556 & AMS \\
\hline Felsstalle & $\mathrm{DE}$ & H-7393-7428 & 12,680 & 120 & 15,565 & 14,230 & \\
\hline Flageolet & FR & Ly-916 & 12,870 & 390 & 16,750 & 14,113 & \\
\hline Fontanet & FR & Ly-846 & 13,810 & 740 & 18,665 & 14,541 & \\
\hline Gare de Couze & FR & BM-1616 & 12,540 & 75 & 15,119 & 14,219 & \\
\hline Gnirshohle 2 & $\mathrm{DE}$ & H-6272-5831 & 13,050 & 300 & 16,823 & 14,667 & \\
\hline Gönnersdorf & $\mathrm{DE}$ & OxA-5728 & 12,730 & 130 & 15,892 & 14,240 & AMS \\
\hline Gönnersdorf & $\mathrm{DE}$ & OxA-5730 & 12,790 & 120 & 16,082 & 14,602 & AMS \\
\hline Goutte Roffat & FR & Ly-3093 & 12,720 & 180 & 16,089 & 14,165 & \\
\hline Hohle Fels, Hohler Fels & $\mathrm{DE}$ & H-5312-4907 & 12,770 & 110 & 15,930 & 14,580 & \\
\hline Jean-Pierre II & FR & Ly-829 & 12,720 & 230 & 16,305 & 14,131 & \\
\hline Kesslerloch & $\mathrm{CH}$ & KIA-33350 & 12,225 & 45 & 14,504 & 13,875 & AMS \\
\hline Kohlerhöhle & $\mathrm{CH}$ & B-4969 & 12,820 & 160 & 16,379 & 14,581 & \\
\hline La Madeleine & FR & Ly-920 & 12,750 & 240 & 16,376 & 14,158 & \\
\hline Labastide, La Grande Grotte & FR & Gif-6367 & 12,700 & 110 & 15,615 & 14,240 & \\
\hline Moulin de Laguenay, Poissière & FR & Ly-3650 & 13,330 & 480 & 17,521 & 14,267 & \\
\hline Oisy & FR & Beta-160913 & 12,500 & 120 & 15,125 & 14,131 & \\
\hline Pellebit & FR & Ly-7003 & 12,610 & 60 & 15,210 & 14,256 & \\
\hline Petersfels & $\mathrm{DE}$ & H-7137-7067 & 12,470 & 100 & 15,077 & 14,132 & \\
\hline Petersfels & $\mathrm{DE}$ & H-7134-6876 & 12500 & 120 & 15,125 & 14,131 & \\
\hline Petersfels & $\mathrm{DE}$ & $\mathrm{H}-7140-7058$ & 12,530 & 90 & 15,119 & 14,191 & \\
\hline Petersfels & $\mathrm{DE}$ & H-7133-6877 & 12,580 & 130 & 15,232 & 14,126 & \\
\hline Petersfels & $\mathrm{DE}$ & H-6653-6786 & 12,600 & 100 & 15,205 & 14,206 & \\
\hline Petersfels & $\mathrm{DE}$ & H-7144-7302 & 12,630 & 95 & 15,415 & 14,206 & \\
\hline Petersfels & $\mathrm{DE}$ & H-4277-3531 & 12,650 & 100 & 15,470 & 14,230 & \\
\hline Petersfels & $\mathrm{DE}$ & H-7214-7350 & 12,660 & 100 & 15,503 & 14,238 & \\
\hline Petersfels & $\mathrm{DE}$ & H-7217-7364 & 12,670 & 90 & 15,526 & 14,258 & \\
\hline Petersfels & $\mathrm{DE}$ & H-7135-6879 & 12,670 & 100 & 15,533 & 14,244 & \\
\hline Petersfels & $\mathrm{DE}$ & H-7141-6985 & 12,680 & 110 & 15,561 & 14,240 & \\
\hline
\end{tabular}


Table 6 (continued)

\begin{tabular}{|c|c|c|c|c|c|c|c|}
\hline Site name & Country & Lab ID & Date & Uncertainty & From cal BP & To cal BP & Type \\
\hline Petersfels & $\mathrm{DE}$ & H-7138-7057 & 12,685 & 75 & 15,524 & 14,573 & \\
\hline Petersfels & $\mathrm{DE}$ & H-7145-7303 & 12,700 & 100 & 15,605 & 14,258 & \\
\hline Pincevent, La Grande-Paroisse & FR & GrN-5760 & 12,545 & 120 & 15,165 & 14,155 & \\
\hline Poggenwisch D & $\mathrm{DE}$ & GrN-11254 & 12,460 & 60 & 15,020 & 14,160 & \\
\hline Poggenwisch D & $\mathrm{DE}$ & $\mathrm{KN}-2754$ & 12,470 & 95 & 15,070 & 14,138 & \\
\hline Poggenwisch D & $\mathrm{DE}$ & $\mathrm{K}-4332$ & 12,570 & 115 & 15,185 & 14,171 & \\
\hline Poggenwisch D & $\mathrm{DE}$ & $\mathrm{H}-136 / 116$ & 12,980 & 370 & 16,800 & 14,241 & \\
\hline Pont d'Ambon & FR & Gif-3369 & 12,840 & 220 & 16,508 & 14,245 & \\
\hline Portel & FR & Gif-2943 & 12,760 & 170 & 16,163 & 14,224 & \\
\hline Quéroy & FR & Gif-5325 & 12,590 & 140 & 15,446 & 14,116 & \\
\hline Quéroy & FR & Gif-5324 & 12,800 & 140 & 16,254 & 14,573 & \\
\hline Roberthill & UK & Q-643 & 12,940 & 250 & 16,690 & 14,577 & \\
\hline Rochedane & FR & Ly-709 & 12,420 & 75 & 15,002 & 14,108 & \\
\hline Rond du Barry & FR & Gif-3492 & 12,800 & 170 & 16,386 & 14,266 & \\
\hline Saaleck & $\mathrm{DE}$ & $\mathrm{KN}-2562$ & 13,200 & 400 & 17,082 & 14,522 & \\
\hline Stellmoor D & $\mathrm{DE}$ & $\mathrm{KN}-2223$ & 12,590 & 80 & 15,180 & 14,238 & \\
\hline Sun Hole & UK & OxA-18705 & 12,490 & 45 & 15,048 & 14,200 & AMS \\
\hline Sun Hole & UK & OxA-14477 & 12,540 & 75 & 15,119 & 14,219 & AMS \\
\hline Sun Hole & UK & OxA-14438 & 12,545 & 55 & 15,125 & 14,237 & AMS \\
\hline Sun Hole & UK & OxA-14476 & 12,610 & 90 & 15,206 & 14,231 & AMS \\
\hline Sun Hole & UK & OxA-19557 & 12,620 & 50 & 15,219 & 14,524 & AMS \\
\hline Teufelsbrücke & $\mathrm{DE}$ & Bln-1727 & 12,480 & 90 & 15,074 & 14,151 & \\
\hline Tournal (or Grande Grotte de Bize) & FR & Ly-1894 & 12,860 & 320 & 16,642 & 14,186 & \\
\hline Trou Da Somme & $\mathrm{BE}$ & OxA-4199 & 12,240 & 130 & 14,898 & 13,814 & AMS \\
\hline Trou de Chaleux & $\mathrm{BE}$ & Lv-1136 & 12,710 & 150 & 15,882 & 14,197 & \\
\hline Vache & FR & GrN-2025 & 12,540 & 105 & 15,140 & 14,179 & \\
\hline Vache, Salle Monique & FR & Gif-7603 & 12,800 & 140 & 16,254 & 14,573 & \\
\hline Verberie, Le Buisson Campin & FR & GifA-99106 & 12,520 & 120 & 15,140 & 14,145 & AMS \\
\hline
\end{tabular}

The lack of dramatic increase in number of dated sites during this phase suggests that northeast and northern expansion began during the warming trend prior to GRIP GI-1e rather than at and after the temperature peak at $14.67 \mathrm{ka}$ cal BP.

\subsection{1-13.9 ka cal BP-GI-1d (Oldest Dryas [Litt et al., 2001] or traditional Older Dryas (Fig. 2f)) (41 dates, 35 sites)}

The Hamburgian is well-represented in northern (Poggenwisch B, Stellmoor D and Querenstede) (Weber and Grimm, 2009) while the Federmesser group is represented by Andernach in the Rhineland and Usselo in the Netherlands. Swiss sites during this phase are attributed to the Azilian. The early Azilian site Le Closeau in the Paris Basin has also produced dates around 14,200-14,800 cal BP (Bodu and Mevel, 2008). Persistence of the Magdalenian in the Paris Basin, the Meuse Basin in Belgium and the northern Rhineland shows some contemporaneity with such new, more northern, groups. In Belgium, occupations at Trou des Blaireaux and Trou Jadot were attributed to the Creswellian, but the existence of this technocomplex in Belgium has been questioned by Charles (Charles, 1996) and more recently has been considered rather a variant of the Final Magdalenian. In central and southwest France, the number of dated sites decreases significantly, but this may not necessarily reflect population decline, which is unlikely given the warmer climate south of $47^{\circ} \mathrm{N}$ than in northwest Europe, where populations survive during this phase. However, the number of dated sites becomes much denser in the next phase (see below). Excluded dates include St. Bees in England (non-archaeological plant material dated in the 1970s, Coope and Joachim, 1980.) and Sun Hole (date obtained in the 1970s).

4.7. 13.9-12.9 ka cal BP - GI-1c to GI-1b (Bølling-Allerød [Litt et al., 2001] or traditional Allerød) (250 dates, 130 sites)

All of the regions previously discussed are now thoroughly occupied: central and southwest France, the Paris Basin, the
Meuse Basin in Belgium, the northern Rhineland and southern Germany (Fig. 2g). In addition, sites are now found in Great Britain (the northernmost being Seamer Carr, Conneller, 2007) and northern Denmark (Norre Lyngby). Pincevent persists through this period and is an example of a well-established seasonally occupied camp. Magdalenian base camps in the Paris Basin and the northern Rhineland thus appear to have been established during the warming trend prior to $14.67 \mathrm{ka}$ cal BP, but do not all persist through GI-1c. Three dates for Gönnersdorf falling within this phase $(11,830 \pm 110$ BP, OxA-2069; $12,380 \pm 230 \mathrm{BP}$, Ly-768; 12,660 $\pm 370 \mathrm{BP}$, Ly-1172) have been rejected (see Stevens et al., 2009, Table 1, p. 134). The Hamburgian also appeared during GI-1e in northern Germany, coexisting with the Northwest Magdalenian.

\subsection{9-11.5 ka cal BP - GI-1a and GS-1 (final Allerød and Younger Dryas [Litt et al., 2001]) (202 dates, 118 sites)}

Site density (Fig. 2h) increases in northern Europe, including Great Britain, but these are no longer Magdalenian, although the Trou du Frontal in Belgium has been attributed to the Magdalenian despite its late date. Discussion of these Final Paleolithic cultures is beyond the scope of this paper.

\section{The Northwest Magdalenian}

The origins of the Northwest Magdalenian can be clearly traced back to southwest and central France, regions with continuity in human occupation through the LGM. These populations may have been demographically increased due to southern migration of northern Gravettian populations at the onset of the LGM. Conversely, the northern populations may have simply decreased in number and become locally extinct. If so, groups repopulating northwest Europe after the LGM would have had genetic continuity with modern populations in the southern latitudes, but none with the northern Early Upper Paleolithic populations. 
Table 7

Dates for 14.1-13.9 ka cal BP.

\begin{tabular}{|c|c|c|c|c|c|c|c|}
\hline Site name & Country & Lab ID & Date & Uncertainty & From cal BP & To cal BP & Type \\
\hline Andernach-Martinsberg 2 & $\mathrm{DE}$ & OxA-V-2218-39 & 12,270 & 50 & 14,832 & 13,928 & AMS \\
\hline Belvis & FR & SacA-6971 & 12,330 & 50 & 14,891 & 14,019 & \\
\hline Buttenloch & $\mathrm{CH}$ & B-4185 & 12,330 & 60 & 14,890 & 14,015 & \\
\hline Cabones & FR & Ly-2296 & 12,620 & 250 & 16,136 & 13,970 & \\
\hline Champréveyres & $\mathrm{CH}$ & UZ-2287 & 12,500 & 150 & 15,163 & 14,064 & AMS \\
\hline Chenelaz & FR & Ly-4790 & 12,610 & 200 & 15,860 & 14,024 & \\
\hline Coléoptère & $\mathrm{BE}$ & Lv-717 & 12,400 & 110 & 15,024 & 14,039 & \\
\hline Conty & FR & Ly-6998 & 12,370 & 70 & 14,937 & 14,048 & \\
\hline Dufaure & FR & Ly-3591 & 12,690 & 230 & 16,225 & 14,075 & \\
\hline Etiolles, Les Coudrays & FR & OxA-12019 & 12,315 & 55 & 14,889 & 13,994 & AMS \\
\hline Etiolles, Les Coudrays & FR & OxA-8757 & 12,315 & 75 & 14,896 & 13,979 & AMS \\
\hline Felsstalle & $\mathrm{DE}$ & $\mathrm{H}-7382-7419$ & 12,320 & 70 & 14,894 & 13,993 & \\
\hline Felsstalle & $\mathrm{DE}$ & H-7392-7428 & 12,400 & 145 & 15,082 & 13,997 & \\
\hline Fontanet & FR & Ly-2184 & 12,770 & 420 & 16,715 & 13,960 & \\
\hline Gönnersdorf & $\mathrm{DE}$ & OxA-15296 & 12,385 & 65 & 14,949 & 14,071 & AMS \\
\hline Hohlenstein-Stadel & $\mathrm{DE}$ & ЕTH-2878 & 12,400 & 180 & 15,129 & 13,926 & AMS \\
\hline La Fru & FR & Ly-2911 & 12,690 & 380 & 16,580 & 13,914 & \\
\hline La Madeleine & FR & Ly-919 & 12,640 & 260 & 16,236 & 13,991 & \\
\hline Laugerie-Basse (Les Marseilles) & FR & Gif-5386 & 12,590 & 250 & 16,060 & 13,927 & \\
\hline Meiendorf D & $\mathrm{DE}$ & $\mathrm{K}-4329$ & 12,360 & 110 & 15,001 & 13,999 & \\
\hline Munzingen & $\mathrm{DE}$ & OxA-4787 & 12,370 & 100 & 14,991 & 14,021 & AMS \\
\hline Petersfels & $\mathrm{DE}$ & $\mathrm{H}-7136-6890$ & 12,320 & 90 & 14,930 & 13,965 & \\
\hline Petersfels & $\mathrm{DE}$ & KN-2884 & 12,400 & 95 & 15,005 & 14,055 & \\
\hline Petersfels & $\mathrm{DE}$ & H-6651-6780 & 12,400 & 100 & 15,010 & 14,050 & \\
\hline Petersfels & $\mathrm{DE}$ & $\mathrm{KN}-2883$ & 12,400 & 100 & 15,010 & 14,050 & \\
\hline Pincevent, La Grande-Paroisse & FR & OxA-148 & 12,600 & 200 & 15,850 & 14,013 & AMS \\
\hline Pont de Longues & FR & Beta-124232 & 12,290 & 60 & 14,875 & 13,943 & \\
\hline Querenstede & $\mathrm{DE}$ & KN-2707 & 12,650 & 320 & 16,421 & 13,943 & \\
\hline Rheinfelden Eremitage & $\mathrm{CH}$ & B-4262 & 12,520 & 170 & 15,246 & 13,994 & \\
\hline Solutré, P16 & FR & Ly-393 & 12,580 & 250 & 16,052 & 13,907 & \\
\hline St. Bees & UK & BIRM-378 & 12,560 & 170 & 15,475 & 14,038 & \\
\hline Stellmoor D & $\mathrm{DE}$ & KN-2224 & 12,530 & 160 & 15,225 & 14,033 & \\
\hline Sun Hole & UK & BM-524 & 12,378 & 150 & 15,071 & 13,962 & \\
\hline Teufelsbrücke & $\mathrm{DE}$ & Bln-1821 & 12,300 & 85 & 14,898 & 13,941 & \\
\hline Teufelsbrücke & $\mathrm{DE}$ & Bln-1924 & 12,315 & 100 & 14,940 & 13,945 & \\
\hline Tournal (or Grande Grotte de Bize) & FR & Ly-1231 & 12,550 & 210 & 15,615 & 13,937 & \\
\hline Trou de Chaleux & $\mathrm{BE}$ & LV-1568 & 12,370 & 170 & 15,090 & 13,917 & \\
\hline Trou des Blaireaux & $\mathrm{BE}$ & LV-1386 & 12,440 & 180 & 15,165 & 13,955 & \\
\hline Trou Jadot & $\mathrm{BE}$ & Lv-1412D & 12,610 & 260 & 16,153 & 13,942 & \\
\hline Tureau des Gardes & FR & Ly-6988 & 12,290 & 90 & 14,893 & 13,920 & \\
\hline Usselo & $\mathrm{NE}$ & Y-139-1 & 12,500 & 180 & 15,242 & 13,953 & \\
\hline Verberie, Le Buisson Campin & FR & GifA-95453 & 12,430 & 120 & 15,062 & 14,058 & AMS \\
\hline Zigeunerfels & $\mathrm{DE}$ & $\mathrm{KN}-3472$ & 12,690 & 280 & 16,381 & 14,039 & \\
\hline
\end{tabular}

While the radiocarbon data provide only a partial picture of Magdalenian expansion, considering the abundance of undated sites that are technologically and typologically Magdalenian as well as sites dated by other means (e.g., TL), mapping the radiocarbon dated sites demonstrates the effects of climatic constraints on northern expansion. Sites are only found south of $47^{\circ} \mathrm{N}$ latitude until the gradual warming trend at the end of the GS-2 (Fig. 3). Then, expansion from southwest to northeast leads to the colonization principally of southern Germany to Poland, with rarer sites in Belgium (Grotte Walou, Sy Verlaine, Chaleux, Trou Da Somme, Frontal) and central Germany (Kniegrotte). Both the Paris Basin and the northern Rhineland show evidence of settlement sites (Etiolles, Verberie, Gönnersdorf, Andernach). Evidence (discussed below) suggests contacts or seasonal movements between both the Paris Basin and the northern Rhineland with the Belgian territory. The Northwest Magdalenian is well-established by the GI-1e, with the additional appearance of the Hamburgian in northern Germany. Population expansion continues northward during GI-1c and $1 \mathrm{~b}$, reaching Great Britain and Denmark, the range of technocomplexes broadening. By the final phase of the Lateglacial, GI-1a and GS-1, northwest Europe is entirely occupied by this range of cultures.

Of interest in Belgium is the absence of Magdalenian sites in the section of the Meuse between Namur and Liège, apart from Goyet on the Samson River, a tributary of the Meuse near Namur. Belgian territory may have been exploited by two different Magdalenian groups, one based in the Paris Basin and the other in the Rhineland. According to this hypothesis, the western Belgian cluster would ultimately be associated with the Paris Basin group, and the eastern sites purely with the northern Rhineland group. The lack of sites between the two, in a cave-rich area long exploited during the Middle and Early Upper Paleolithic, suggests that the territories exploited during the Magdalenian did not overlap (Fig. 4). Alternatively, the density of Magdalenian sites in Belgian territory may indicate an intermediate territory having contacts with both the Paris basin and the northern Rhineland.

Several lines of evidence point to connections between the Paris Basin and Belgian sites along the Meuse and on the Hesbaye Plateau. De Bie and Caspar (1994, p. 145), discussing burin technology in the context of the Federmesser site of Rekem, point out a significant correlation between burin facet width and burin type at Orp (Vermeersch et al., 1987: 40) and Le Grand Canton (Valentin, 1995: 231). Wenzel (2005, p. 25) points out contemporaneity and similarity in lithic assemblage structure between Orp, Unit U5, and the site of Etiolles. Fossil marine shells from the Paris Basin are found at Belgian sites (Chaleux, Trou Da Somme, Bois Laiterie, Sy Verlaine) (Dewez, 1987; Taborin, 1993; Lozouet and Gautier, 1997) (Fig. 5). Silicified limestone, from a primary source near CharlevilleMaizière, was transported downstream along the Meuse to the 
Table 8

Dates for 13.9-12.9 ka cal BP.

\begin{tabular}{|c|c|c|c|c|c|c|c|}
\hline Site name & Country & Lab ID & Date & Uncertainty & From cal BP & To cal BP & Type \\
\hline Abri du Mannlefelsen, & FR & Lv-1144 & 11,760 & 120 & 13,854 & 13,352 & \\
\hline Abri Gay & FR & LYON-640 & 12,160 & 60 & 14,190 & 13,819 & AMS \\
\hline Abri Martin & FR & MC-2348 & 12,000 & 250 & 14,889 & 13,358 & \\
\hline Abri nord de Bavans & FR & Gif-6061 & 12,220 & 170 & 14,969 & 13,761 & \\
\hline Abri Pataud & FR & Grn-2079 & 11,320 & 110 & 13,413 & 12,932 & \\
\hline Adaouste & FR & LGQ-126 & 12,054 & 375 & 15,435 & 13,141 & \\
\hline Adaouste & FR & Ly-541 & 12,280 & 190 & 15,046 & 13,797 & \\
\hline Adaouste & FR & LGQ-125 & 12,497 & 374 & 16,393 & 13,722 & \\
\hline Adaouste & FR & LGQ-374 & 12,497 & 374 & 16,393 & 13,722 & \\
\hline Andernach-Martinsberg & $\mathrm{DE}$ & OxA-999 & 12,500 & 500 & 16,603 & 13,500 & AMS \\
\hline Andernach-Martinsberg 2 & $\mathrm{DE}$ & OxA-997 & 11,800 & 160 & 13,980 & 13,325 & AMS \\
\hline Andernach-Martinsberg 2 & $\mathrm{DE}$ & GrA-16990 & 11,820 & 70 & 13,830 & 13,451 & AMS \\
\hline Andernach-Martinsberg 2 & $\mathrm{DE}$ & OxA-1924 & 11,890 & 120 & 13,997 & 13,441 & AMS \\
\hline Andernach-Martinsberg 2 & $\mathrm{DE}$ & OxA-984 & 11,950 & 250 & 14,857 & 13,296 & AMS \\
\hline Andernach-Martinsberg 2 & $\mathrm{DE}$ & GrA-16989 & 11,960 & 70 & 14,006 & 13,634 & AMS \\
\hline Andernach-Martinsberg 2 & $\mathrm{DE}$ & GrA-16991 & 12,040 & 70 & 14,073 & 13,733 & AMS \\
\hline Andernach-Martinsberg 2 & $\mathrm{DE}$ & GrA-16987 & 12,050 & 70 & 14,086 & 13,742 & AMS \\
\hline Andernach-Martinsberg 2 & $\mathrm{DE}$ & OxA-985 & 12,300 & 200 & 15,078 & 13,802 & AMS \\
\hline Bange & FR & Ly-3640 & 11,680 & 190 & 13,925 & 13,181 & \\
\hline Baume de Gigny & FR & Ly-1798 & 12,370 & 460 & 16,401 & 13,416 & \\
\hline Baume de Vallorgues & FR & KN-1068 & 11,620 & 110 & 13,740 & 13,272 & \\
\hline Baume de Vallorgues & FR & Hv-1346 & 12,060 & 250 & 14,953 & 13,413 & \\
\hline Baume de Vallorgues & FR & Kn-67 & 12,060 & 250 & 14,953 & 13,413 & \\
\hline Baume Goulon & FR & & 11,865 & 70 & 13,878 & 13,471 & \\
\hline Bettenroder Berg IX & $\mathrm{DE}$ & Hv-17371 & 11,770 & 350 & 14,903 & 12,949 & \\
\hline Birseck-Ermitage & $\mathrm{CH}$ & B-4260 & 11,860 & 100 & 13,926 & 13,442 & \\
\hline Birseck-Ermitage & $\mathrm{CH}$ & B-4261 & 12,040 & 80 & 14,108 & 13,725 & \\
\hline Bourrouilla, Grotte d'Arancou & FR & Gif-10002 & 12,260 & 120 & 14,905 & 13,842 & \\
\hline Breitenbach B & $\mathrm{DE}$ & KN-3620 & 12,320 & 200 & 15,090 & 13,819 & \\
\hline Brohltal & $\mathrm{DE}$ & Hd-17100 & 11,206 & 20 & 13,244 & 12,930 & \\
\hline Brohltal & $\mathrm{DE}$ & Hd-17145 & 11,223 & 22 & 13,275 & 12,956 & \\
\hline Brohltal & $\mathrm{DE}$ & Hd-17900 & 11,277 & 26 & 13,278 & 13,101 & \\
\hline Brohltal & $\mathrm{DE}$ & KN-3802 & 11,280 & 100 & 13,367 & 12,907 & \\
\hline Brohltal & $\mathrm{DE}$ & KN-3803 & 11,510 & 90 & 13,598 & 13,178 & \\
\hline Bruniquel, Bruniquet & FR & BM-304 & 12,070 & 180 & 14,846 & 13,461 & \\
\hline Budel II-2 & NE & GrN-1675 & 11,440 & 120 & 13,591 & 13,098 & \\
\hline Buttenloch & $\mathrm{CH}$ & B-4187 & 12,090 & 70 & 14,129 & 13,774 & \\
\hline Buttenloch & $\mathrm{CH}$ & B-4186 & 12,170 & 50 & 14,185 & 13,843 & \\
\hline Cabones & FR & Ly-3079 & 11,520 & 190 & 13,815 & 12,980 & \\
\hline Cabones & FR & Gif-8396 & 12,150 & 80 & 14,465 & 13,766 & \\
\hline Canecaude & FR & Gif-3448 & 12,300 & 600 & 16,655 & 13,205 & \\
\hline Chabot & FR & Ly-697 & 12,000 & 410 & 15,517 & 13,085 & \\
\hline Chauveau & $\mathrm{BE}$ & Lv-1961 & 12,000 & 130 & 14,165 & 13,484 & \\
\hline Chez Jugie & FR & Ly-1802 & 13,000 & 1000 & 18,495 & 13,263 & \\
\hline Chinchon I & FR & Ly-597 & 12,000 & 420 & 15,570 & 12,985 & \\
\hline Coléoptère & $\mathrm{BE}$ & Lv-686 & 12,150 & 150 & 14,876 & 13,704 & \\
\hline Colle Rousse & FR & Gif-8657 & 12,330 & 150 & 15,028 & 13,898 & \\
\hline Colombier & FR & Ly-4811 & 12,150 & 240 & 15,029 & 13,499 & \\
\hline Conduché & FR & Ly-2693 & 12,040 & 160 & 14,542 & 13,454 & \\
\hline Conty & FR & Ly-285 & 11,420 & 65 & 13,420 & 13,142 & \\
\hline Conty & FR & LYON-1353 & 11,885 & 75 & 13,914 & 13,480 & \\
\hline Coufin & FR & Ly-2930 & 11,590 & 190 & 13,836 & 13,113 & \\
\hline Courbet & FR & BM-302 & 11,750 & 300 & 14,607 & 12,951 & \\
\hline Deux-Avens & FR & Ly-321 & 12,320 & 600 & 16,675 & 13,235 & \\
\hline Deux-Avens & FR & Ly-322 & 12,350 & 200 & 15,114 & 13,841 & \\
\hline Douattes & FR & Ly-435 & 12,480 & 260 & 15,846 & 13,789 & \\
\hline Doue & FR & Ly-2822 & 11,520 & 170 & 13,767 & 13,098 & \\
\hline Dufaure & FR & Ly-3181 & 11,750 & 300 & 14,607 & 12,951 & \\
\hline Dufaure & FR & Ly-3245 & 12,030 & 280 & 14,990 & 13,355 & \\
\hline Dufaure & FR & Ly-3182 & 12,260 & 400 & 16,046 & 13,320 & \\
\hline Dufaure & FR & Ly-2923 & 12,290 & 270 & 15,235 & 13,504 & \\
\hline Dumas (Saut du Loup) & FR & Ly-318 & 11,750 & 300 & 14,607 & 12,951 & \\
\hline Dumas (Saut du Loup) & FR & Ly-319 & 12,080 & 310 & 15,104 & 13,349 & \\
\hline Duruthy & FR & BOR7 & 14,500 & 1450 & 21,727 & 13,797 & \\
\hline Espelugues, Le Calvaire CPE Gauche & FR & Ly-1906 & 12,450 & 330 & 16,139 & 13,689 & \\
\hline Etiolles, Les Coudrays & FR & OxA-174 & 11,900 & 250 & 14,823 & 13,250 & AMS \\
\hline Etiolles, Les Coudrays & FR & Ly-1351 & 12,000 & 220 & 14,850 & 13,375 & \\
\hline Etiolles, Les Coudrays & FR & OxA-5995/LYON-202 & 12,250 & 100 & 14,878 & 13,841 & AMS \\
\hline Etrembieres & FR & B-3787 & 12,310 & 140 & 14,995 & 13,885 & \\
\hline Fanciulli, Grotte des Enfants, Baousse Rousse & FR & MC-499 & 12,200 & 400 & 15,883 & 13,279 & \\
\hline Fantas & FR & Ny-128 & 12,490 & 270 & 15,869 & 13,795 & \\
\hline Faurelie & FR & Gif-3649 & 11,780 & 180 & 14,006 & 13,286 & \\
\hline Faustin & FR & Ly-2700 & 12,370 & 220 & 15,172 & 13,806 & \\
\hline Felsstalle & $\mathrm{DE}$ & $\mathrm{H}-7394-7429$ & 11,800 & 200 & 14,099 & 13,264 & \\
\hline
\end{tabular}


Table 8 (continued)

\begin{tabular}{|c|c|c|c|c|c|c|c|}
\hline Site name & Country & Lab ID & Date & Uncertainty & From cal BP & To cal BP & Type \\
\hline Felsstalle & $\mathrm{DE}$ & H-7391-7426 & 12,050 & 115 & 14,220 & 13,599 & \\
\hline Felsstalle & $\mathrm{DE}$ & H-7383-7419 & 12,100 & 60 & 14,124 & 13,790 & \\
\hline Gare de Conduche & FR & Ly-3693 & 12,040 & 160 & 14,542 & 13,454 & \\
\hline Gare de Couze & FR & Ly-976 & 11,750 & 310 & 14,807 & 12,946 & \\
\hline Gare de Couze & FR & Ly-975 & 12,430 & 320 & 16,056 & 13,662 & \\
\hline Gay & FR & Ly-725 & 11,660 & 240 & 14,072 & 13,083 & \\
\hline Gnirshohle 2 & $\mathrm{DE}$ & H-6270-5832 & 12,360 & 200 & 15,121 & 13,849 & \\
\hline Gnirshohle 2 & $\mathrm{DE}$ & H-6271-5830 & 12,600 & 320 & 16,349 & 13,878 & \\
\hline Gönnersdorf & $\mathrm{DE}$ & OxA-2069 & 11,830 & 110 & 13,910 & 13,415 & AMS \\
\hline Gönnersdorf & $\mathrm{DE}$ & Ly-768 & 12,380 & 230 & 15,218 & 13,772 & \\
\hline Gönnersdorf & $\mathrm{DE}$ & Ly-1172 & 12,660 & 370 & 16,525 & 13,885 & \\
\hline Gough's New Cave & UK & BM-2187 & 12,070 & 170 & 14,831 & 13,465 & \\
\hline Gough's New Cave & UK & BM-2183 & 12,120 & 120 & 14,558 & 13,689 & \\
\hline Gough's New Cave & UK & BM-2185R & 12,200 & 250 & 15,117 & 13,582 & \\
\hline Gough's New Cave & UK & BM-2184R & 12,250 & 160 & 14,967 & 13,798 & \\
\hline Gough's New Cave & UK & BM-2186R & 12,470 & 240 & 15,553 & 13,820 & \\
\hline Goutte Roffat & FR & Ly-3096 & 11,940 & 280 & 14,898 & 13,275 & \\
\hline Goutte Roffat & FR & Ly-3095 & 12,090 & 170 & 14,849 & 13,492 & \\
\hline Goutte Roffat & FR & Ly-3098 & 12,330 & 300 & 15,561 & 13,500 & \\
\hline Goutte Roffat & FR & Ly-3094 & 12,420 & 320 & 16,049 & 13,637 & \\
\hline Goyet & $\mathrm{BE}$ & Lv-2135 & 11,630 & 150 & 13,805 & 13,220 & \\
\hline Grand Canton & FR & Gif-9609 & 11,420 & 100 & 13,473 & 13,103 & \\
\hline Grand Canton & FR & Gif-9607 & 12,080 & 115 & 14,483 & 13,633 & \\
\hline Grand Canton & FR & Gif-9606 & 12,195 & 130 & 14,874 & 13,766 & \\
\hline Graves & FR & Gif-7340 & 11,360 & 120 & 13,463 & 12,945 & \\
\hline Grotte du Cheval & FR & Gif-92346 & 12,050 & 130 & 14,480 & 13,489 & \\
\hline High Furlong & UK & St-3836 & 11,665 & 140 & 13,811 & 13,267 & \\
\hline High Furlong & UK & St-3832 & 12,200 & 160 & 14,932 & 13,751 & \\
\hline Jean Pierre II & FR & Ly-925 & 12,400 & 240 & 15,447 & 13,764 & \\
\hline Jean Pierre II & FR & Ly-828 & 12,470 & 200 & 15,413 & 13,873 & \\
\hline Jean-Pierre I & FR & Ly-627 & 11,700 & 220 & 14,019 & 13,140 & \\
\hline Jean-Pierre I & FR & Ly-429 & 11,900 & 360 & 15,071 & 13,114 & \\
\hline Jean-Pierre II & FR & Ly-693 & 11,630 & 240 & 14,036 & 12,981 & \\
\hline Kastelhöhle & $\mathrm{CH}$ & B-4637 & 11,380 & 80 & 13,403 & 13,110 & \\
\hline Kastelhöhle & $\mathrm{CH}$ & B-4253 & 11,389 & 150 & 13,605 & 12,918 & \\
\hline Kastelhöhle & $\mathrm{CH}$ & B-4255 & 11,680 & 50 & 13,708 & 13,379 & \\
\hline Kastelhöhle & $\mathrm{CH}$ & B-4256 & 12,110 & 60 & 14,136 & 13,796 & \\
\hline Kent's Cavern, Black Band & UK & GrN-6204 & 12,180 & 100 & 14,798 & 13,769 & \\
\hline Klappholz LA 63 & $\mathrm{DE}$ & AAR-2785 & 11,560 & 110 & 13,698 & 13,206 & \\
\hline Klein-Nordende CR & $\mathrm{DE}$ & $\mathrm{Kl}-2152$ & 11,990 & 100 & 14,110 & 13,600 & \\
\hline Klein-Nordende CR & $\mathrm{DE}$ & $\mathrm{Kl}-2124$ & 12,035 & 110 & 14,176 & 13,625 & \\
\hline Kohlerhöhle & $\mathrm{CH}$ & B-4971 & 11,640 & 150 & 13,814 & 13,229 & \\
\hline La Caune & FR & Gif-2950 & 12,270 & 280 & 15,226 & 13,477 & \\
\hline La Fru & FR & Ly-2409 & 11,680 & 150 & 13,840 & 13,262 & \\
\hline La Fru & FR & Ly-4325 & 11,740 & 110 & 13,821 & 13,357 & \\
\hline La Fru & FR & Ly-2250 & 11,810 & 160 & 13,991 & 13,334 & \\
\hline La Fru & FR & Ly-2408 & 11,820 & 230 & 14,465 & 13,160 & \\
\hline La Fru & FR & Ly-2751 & 12,470 & 400 & 16,418 & 13,636 & \\
\hline La Fru & FR & Ly-2915 & 12,680 & 560 & 16,850 & 13,629 & \\
\hline La Garenne Grand Abri & FR & C-579 & 12,986 & 560 & 17,104 & 13,855 & \\
\hline La Goutte Roffat & FR & Ly-3097 & 12,150 & 200 & 14,986 & 13,612 & \\
\hline La Quina & FR & GrN-4450 & 11,690 & 70 & 13,742 & 13,370 & \\
\hline Le Chaumois Boivin & FR & Ly-440 & 12,040 & 270 & 14,981 & 13,377 & \\
\hline Le Marais Gravière III & FR & Ly-22 & 11,660 & 110 & 13,761 & 13,298 & \\
\hline Le Martinet & FR & Ly-1605 & 13,600 & 1100 & 19,276 & 13,622 & \\
\hline L'Eléphant & FR & Gif-8227 & 12,020 & 100 & 14,137 & 13,645 & \\
\hline L'Eléphant & FR & Gif- 8228 & 12,290 & 110 & 14,927 & 13,894 & \\
\hline Limeuil-Village & FR & Gif- 8040 & 11,720 & 110 & 13,805 & 13,340 & \\
\hline Longetraye & FR & Ly-512 & 12,720 & 750 & 17,393 & 13,291 & \\
\hline Lortet & FR & Ly-3437 & 12,300 & 200 & 15,078 & 13,802 & \\
\hline Malarode & FR & Ly-3706 & 12,420 & 280 & 15,841 & 13,715 & \\
\hline Mas-d'Azil & FR & Gif-5679 & 13,400 & 1000 & 18,700 & 13,577 & \\
\hline Meiendorf D & $\mathrm{DE}$ & W-264 & 11,790 & 200 & 14,086 & 13,256 & \\
\hline Meiendorf D & $\mathrm{DE}$ & $\mathrm{W}-281$ & 11,870 & 200 & 14,223 & 13,271 & \\
\hline Meiendorf D & $\mathrm{DE}$ & $\mathrm{H}-38 / 121 \mathrm{~B}$ & 12,300 & 200 & 15,078 & 13,802 & \\
\hline Meiendorf D & $\mathrm{DE}$ & $\mathrm{H}-38 / 121 \mathrm{~A}$ & 12,300 & 300 & 15,492 & 13,478 & \\
\hline Meiendorf D & $\mathrm{DE}$ & $\mathrm{KN}-2220$ & 12,470 & 250 & 15,617 & 13,791 & \\
\hline Miesenheim 2 & $\mathrm{DE}$ & $\mathrm{KN}-3578$ & 11,290 & 80 & 13,349 & 12,957 & \\
\hline Miesenheim 2 & $\mathrm{DE}$ & KN-3517 & 11,290 & 95 & 13,366 & 12,929 & \\
\hline Miesenheim 2 & $\mathrm{DE}$ & KN-3577 & 11,310 & 95 & 13,386 & 12,957 & \\
\hline Miesenheim 2 & $\mathrm{DE}$ & KN-3535 & 11,330 & 100 & 13,415 & 12,960 & \\
\hline Miesenheim 2 & $\mathrm{DE}$ & KN-3534 & 11,360 & 110 & 13,455 & 12,965 & \\
\hline Miesenheim 2 & $\mathrm{DE}$ & KN-3529 & 11,370 & 85 & 13,409 & 13,100 & \\
\hline Miesenheim 2 & $\mathrm{DE}$ & KN-3580 & 11,370 & 100 & 13,456 & 13,076 & \\
\hline Miesenheim 2 & $\mathrm{DE}$ & KN-3532 & 11,390 & 90 & 13,429 & 13,105 & \\
\hline Miesenheim 2 & $\mathrm{DE}$ & KN-3581 & 11,440 & 100 & 13,498 & 13,107 & \\
\hline
\end{tabular}


Table $\mathbf{8}$ (continued)

\begin{tabular}{|c|c|c|c|c|c|c|c|}
\hline Site name & Country & Lab ID & Date & Uncertainty & From cal BP & To cal BP & Type \\
\hline Miesenheim 2 & $\overline{\mathrm{DE}}$ & KN-3533 & 11,460 & 90 & 13,496 & 13,125 & \\
\hline Miesenheim 2 & $\mathrm{DE}$ & KN-3531 & 11,460 & 100 & 13,564 & 13,117 & \\
\hline Miesenheim 2 & $\mathrm{DE}$ & KN-3575 & 11,460 & 100 & 13,564 & 13,117 & \\
\hline Miesenheim 2 & $\mathrm{DE}$ & KN-3530 & 11,470 & 100 & 13,567 & 13,127 & \\
\hline Milheeze-Hogeloop & $\mathrm{NE}$ & GrN-16509 & 11,445 & 35 & 13,419 & 13,199 & \\
\hline Mollendruz-Abri Freymond & $\mathrm{CH}$ & ETH-5600 & 11,760 & 145 & 13,900 & 13,315 & AMS \\
\hline Mollendruz-Abri Freymond & $\mathrm{CH}$ & ETH-5601 & 12,020 & 120 & 14,185 & 13,503 & AMS \\
\hline Mollendruz-Abri Freymond & $\mathrm{CH}$ & OxA-9640 & 12,780 & 80 & 15,846 & 14,715 & AMS \\
\hline Montgaudier & FR & BM-1912 & 12,180 & 130 & 14,865 & 13,751 & \\
\hline Montgaudier Abri Gaudry & FR & BM-1911 & 11,450 & 70 & 13,450 & 13,155 & \\
\hline Montgaudier Abri Gaudry & FR & BM-2308 & 11,930 & 190 & 14,479 & 13,323 & \\
\hline Moosbuhl & $\mathrm{CH}$ & B-2310 & 11,900 & 130 & 14,025 & 13,434 & \\
\hline Moosbuhl & $\mathrm{CH}$ & B-2316 & 12,060 & 150 & 14,544 & 13,481 & \\
\hline Munzingen & $\mathrm{DE}$ & $\mathrm{H}-4738-4660$ & 12,130 & 95 & 14,486 & 13,741 & \\
\hline Norre Lyngby & DK & AAR-1511 & 11,570 & 110 & 13,711 & 13,224 & \\
\hline Oldeholtwolde & $\mathrm{NE}$ & GrN-11264 & 11,340 & 100 & 13,428 & 12,966 & \\
\hline Oldeholtwolde & NE & GrN-13083 & 11,600 & 250 & 14,009 & 12,947 & \\
\hline Ossom's Cave & UK & BM-2127R & 12,220 & 320 & 15,461 & 13,389 & \\
\hline Oudehaske & $\mathrm{NE}$ & GrN-18784 & 11,390 & 65 & 13,393 & 13,125 & \\
\hline Pégourié & FR & Ly-1833 & 11,850 & 280 & 14,830 & 13,143 & \\
\hline Pégourié & FR & Ly-1832 & 11,870 & 290 & 14,861 & 13,169 & \\
\hline Pégourié & FR & Ly-3851 & 12,160 & 200 & 14,994 & 13,629 & \\
\hline Pégourié & FR & Ly-3852 & 12,160 & 200 & 14,994 & 13,629 & \\
\hline Pégourié & FR & Gif-2822 & 12,250 & 350 & 15,822 & 13,356 & \\
\hline Pégourié & FR & Ly-1392 & 12,690 & 530 & 16,825 & 13,715 & \\
\hline Petersfels & $\mathrm{DE}$ & $\mathrm{H}-4741-4145$ & 11,300 & 85 & 13,368 & 12,959 & \\
\hline Petersfels & $\mathrm{DE}$ & H-4276-3535 & 11,700 & 90 & 13,770 & 13,351 & \\
\hline Petersfels & $\mathrm{DE}$ & H-6650-6779 & 11,700 & 100 & 13,779 & 13,338 & \\
\hline Petersfels & $\mathrm{DE}$ & $\mathrm{H}-4343-4137$ & 11,890 & 130 & 14,015 & 13,430 & \\
\hline Petersfels & $\mathrm{DE}$ & H-5209-4865 & 11,975 & 100 & 14,080 & 13,505 & \\
\hline Petersfels & $\mathrm{DE}$ & H-6654-6787 & 12,100 & 280 & 15,055 & 13,415 & \\
\hline Petersfels & $\mathrm{DE}$ & H-7132-6984 & 12,120 & 100 & 14,489 & 13,729 & \\
\hline Petersfels & $\mathrm{DE}$ & H-5210-4866 & 12,160 & 95 & 14,521 & 13,767 & \\
\hline Petersfels & $\mathrm{DE}$ & H-7139-7300 & 12,180 & 100 & 14,798 & 13,769 & \\
\hline Petersfels & $\mathrm{DE}$ & H-6652-6783 & 12,230 & 240 & 15,120 & 13,645 & \\
\hline Petersfels & $\mathrm{DE}$ & H-7215-7349 & 12,270 & 95 & 14,884 & 13,881 & \\
\hline Petersfels & $\mathrm{DE}$ & H-6655-6788 & 12,500 & 220 & 15,526 & 13,889 & \\
\hline Pincevent, La Grande-Paroisse & FR & Gif-6284 & 11,800 & 130 & 13,922 & 13,371 & \\
\hline Pincevent, La Grande-Paroisse & FR & OxA-391 & 11,870 & 130 & 13,995 & 13,419 & AMS \\
\hline Pincevent, La Grande-Paroisse & FR & OxA-176 & 12,000 & 220 & 14,850 & 13,375 & AMS \\
\hline Pincevent, La Grande-Paroisse & FR & Gif-5971 & 12,100 & 120 & 14,522 & 13,668 & \\
\hline Pincevent, La Grande-Paroisse & FR & Gif-6310 & 12,100 & 130 & 14,565 & 13,624 & \\
\hline Pincevent, La Grande-Paroisse & FR & Gif-6283 & 12,120 & 130 & 14,813 & 13,662 & \\
\hline Pincevent, La Grande-Paroisse & FR & OxA-467 & 12,250 & 160 & 14,967 & 13,798 & AMS \\
\hline Pincevent, La Grande-Paroisse & FR & Erl-6786 & 12,277 & 96 & 14,887 & 13,893 & \\
\hline Pincevent, La Grande-Paroisse & FR & Erl-6786 & 12,277 & 96 & 14,887 & 13,893 & \\
\hline Pincevent, La Grande-Paroisse & FR & OxA-177 & 12300 & 220 & 15,119 & 13,770 & AMS \\
\hline Pincevent, La Grande-Paroisse & FR & Gif-358 & 12,300 & 400 & 16,094 & 13,379 & \\
\hline Pincevent, La Grande-Paroisse & FR & OxA-149 & 12,400 & 200 & 15,159 & 13,872 & AMS \\
\hline Poggenwisch & $\mathrm{DE}$ & GrN-11262 & 11,250 & 50 & 13,298 & 12,961 & \\
\hline Poggenwisch D & $\mathrm{DE}$ & $\mathrm{W}-271$ & 11,750 & 200 & 14,033 & 13,230 & \\
\hline Pont d'Ambon & FR & Gif-7223 & 11,600 & 120 & 13,747 & 13,244 & \\
\hline Pont d'Ambon & FR & Gif-3739 & 12,130 & 160 & 14,876 & 13,645 & \\
\hline Pont de Longues & FR & Beta-108629 & 11,720 & 50 & 13,737 & 13,412 & \\
\hline Pont de Longues & FR & Beta-110898 & 11,920 & 50 & 13,935 & 13,614 & \\
\hline Poyemau or Poeymaü & FR & Ly-1385 & 11,540 & 220 & 13,863 & 12,950 & \\
\hline Poyemau or Poeymaü & FR & Ly-1384 & 12,000 & 250 & 14,889 & 13,358 & \\
\hline Pré de la Chapelle & FR & ETH-15766 & 11,480 & 190 & 13,768 & 12,959 & AMS \\
\hline Pré de la Chapelle & FR & Ly-849 & 11,860 & 190 & 14,148 & 13,303 & \\
\hline Preletang & FR & Ly-93 & 11,730 & 260 & 14,470 & 13,074 & \\
\hline Presles & $\mathrm{BE}$ & LV-1472 & 12,140 & 160 & 14,880 & 13,670 & \\
\hline Quinçay [Grande Roche de la Plematrie] & FR & Ly-790 & 11,910 & 200 & 14,481 & 13,297 & \\
\hline Rheinfelden Eremitage & $\mathrm{CH}$ & B-4183 & 11,600 & 120 & 13,747 & 13,244 & \\
\hline Rheinfelden Eremitage & $\mathrm{CH}$ & B-4184 & 11,950 & 50 & 13,964 & 13,662 & \\
\hline Rhodes II & FR & MC-997 & 12,100 & 150 & 14,846 & 13,595 & \\
\hline Rhodes II & FR & Gif-2258 & 12,160 & 160 & 14,906 & 13,706 & \\
\hline Rhodes II & FR & MC-1366 & 12,250 & 200 & 15,050 & 13,759 & \\
\hline Rhodes II & FR & MC-996 & 12,300 & 150 & 14,996 & 13,860 & \\
\hline Rislisberghöhle & $\mathrm{CH}$ & Ly-1099 & 11,860 & 230 & 14,515 & 13,236 & \\
\hline Rissen $14 \mathrm{a}$ & $\mathrm{NE}$ & $\mathrm{H}-75 / 78$ & 11,450 & 180 & 13,731 & 12,945 & \\
\hline Roc de Marcamp & FR & Ly-3148 & 11,910 & 230 & 14,596 & 13,267 & \\
\hline Roc-aux-Sorciers, Abri Bourdois & FR & & 12,210 & 80 & 14,790 & 13,809 & \\
\hline Rocher de la Caille (Saut-du-Perron) & FR & Ly-5645 & 12,214 & 476 & 16,285 & 13,249 & \\
\hline Romains & FR & MC-1276 & 12,540 & 230 & 15,841 & 13,885 & \\
\hline Romains & FR & MC-1215 & 12,540 & 400 & 16,490 & 13,735 & \\
\hline
\end{tabular}


Table 8 (continued)

\begin{tabular}{|c|c|c|c|c|c|c|c|}
\hline Site name & Country & Lab ID & Date & Uncertainty & From cal BP & To cal BP & Type \\
\hline Romains & FR & MC-1275 & 12,540 & 400 & 16,490 & 13,735 & \\
\hline Rond du Barry & FR & Gif-2671 & 12,380 & 280 & 15,596 & 13,661 & \\
\hline Roquemissou Abri $n^{\circ} 2$ des Usclades & FR & Gif-10313 & 11,400 & 85 & 13,425 & 13,114 & \\
\hline Roquemissou Abri $n^{\circ} 2$ des Usclades & FR & Gif-10312 & 11,590 & 105 & 13,716 & 13,254 & \\
\hline Rosenhof & $\mathrm{DE}$ & $\mathrm{KN}-3196$ & 11,900 & 100 & 13,973 & 13,466 & \\
\hline Rosmos & DK & K-3697 & 13,240 & 760 & 17,986 & 13,791 & \\
\hline Seamer K & UK & CAR-842 & 12,010 & 130 & 14,181 & 13,484 & \\
\hline Soppensee & $\mathrm{CH}$ & ETH-1615 & 13,370 & 900 & 18,526 & 13,784 & AMS \\
\hline St. Bees & UK & BIRM-647 & 11,500 & 120 & 13,641 & 13,130 & \\
\hline Stellmoor D & $\mathrm{DE}$ & $\mathrm{K}-4328$ & 12,180 & 130 & 14,865 & 13,751 & \\
\hline Stellmoor D & $\mathrm{DE}$ & K-4261 & 12,190 & 125 & 14,863 & 13,764 & \\
\hline Stellmoor D & $\mathrm{DE}$ & W-261 & 12,450 & 200 & 15,219 & 13,876 & \\
\hline Taï & FR & Ly-4217 & 11,580 & 180 & 13,808 & 13,121 & \\
\hline Taï & FR & Ly-4218 & 11,980 & 160 & 14,468 & 13,403 & \\
\hline Taï & FR & Ly-3442 & 12,290 & 220 & 15,114 & 13,763 & \\
\hline Trou Jadot & $\mathrm{BE}$ & Lv-1411 & 11,850 & 160 & 14,037 & 13,359 & \\
\hline Tureau des Gardes & FR & Ly-6989 & 11,560 & 100 & 13,681 & 13,224 & \\
\hline Vache & FR & Col-336c & 11,650 & 200 & 13,912 & 13,137 & \\
\hline Verberie, Le Buisson Campin & FR & GifA-99421 & 12,300 & 120 & 14,956 & 13,896 & AMS \\
\hline Weissensee & $\mathrm{DE}$ & KN-3623 & 11,600 & 230 & 13,982 & 12,980 & \\
\hline Westerkappeln, Fundstelle C & $\mathrm{DE}$ & $\mathrm{KI}-271$ & 11,800 & 200 & 14,099 & 13,264 & \\
\hline Zigeunerfels & $\mathrm{DE}$ & $\mathrm{KN}-3478$ & 11,290 & 90 & 13,359 & 12,938 & \\
\hline Zigeunerfels & DE & $\mathrm{KN}-3474$ & 11,300 & 100 & 13,383 & 12,931 & \\
\hline Zigeunerfels & $\mathrm{DE}$ & $\mathrm{KN}-3471$ & 11,480 & 100 & 13,576 & 13,137 & \\
\hline Zigeunerfels & $\mathrm{DE}$ & $\mathrm{KN}-3482$ & 11,830 & 100 & 13,887 & 13,425 & \\
\hline Zigeunerfels & DE & $\mathrm{KN}-3486$ & 11,910 & 100 & 13,984 & 13,473 & \\
\hline Zigeunerfels & $\mathrm{DE}$ & $\mathrm{KN}-3473$ & 12,100 & 100 & 14,460 & 13,702 & \\
\hline Zigeunerfels & $\mathrm{DE}$ & $\mathrm{KN}-3481$ & 12,200 & 600 & 16,597 & 13,104 & \\
\hline
\end{tabular}

Table 9

Dates for $12.9-11.5 \mathrm{ka}$ cal BP.

\begin{tabular}{|c|c|c|c|c|c|c|c|}
\hline Site name & Country & Lab ID & Date & Uncertainty & From cal BP & To cal BP & Type \\
\hline Aardhorst & $\mathrm{NE}$ & GrN-4180 & 11,140 & 70 & 13,210 & 12,766 & \\
\hline Abeurador & FR & Gif-6746 & 10,480 & 100 & 12,605 & 12,075 & \\
\hline Abri du Mannlefelsen, & FR & Lv-1036 & 10,560 & 200 & 12,897 & 11,770 & \\
\hline Abri du Mannlefelsen, & FR & Lv-1090 & 11,080 & 100 & 13,175 & 12,688 & \\
\hline Abri Martin & FR & MC-2345 & 10,200 & 0 & 12,020 & 11,823 & \\
\hline Abri Martin & FR & MC-23464 & 10,820 & 200 & 13,175 & 12,163 & \\
\hline Abri Pataud & FR & GrN-3644 & 10,450 & 120 & 12,619 & 11,978 & \\
\hline Altwasser Höhle 1 & $\mathrm{CH}$ & ETH-9641 & 10,240 & 85 & 12,389 & 11,621 & AMS \\
\hline Andernach-Martinsberg & $\mathrm{DE}$ & GrA-16521 & 10,970 & 60 & 13,070 & 12,665 & AMS \\
\hline Andernach-Martinsberg & $\mathrm{DE}$ & $\mathrm{H}-85 / 91$ & 11,300 & 220 & 13,617 & 12,692 & \\
\hline Andernach-Martinsberg 3 & $\mathrm{DE}$ & GrA-16994 & 11,160 & 70 & 13,245 & 12,800 & AMS \\
\hline Andernach-Martinsberg 3 & $\mathrm{DE}$ & OxA-998 & 11,370 & 160 & 13,615 & 12,879 & AMS \\
\hline Baerenkeller & $\mathrm{DE}$ & B-980 & 11,190 & 180 & 13,382 & 12,679 & \\
\hline Baraquettes & FR & Beta-122220 & 10,170 & 60 & 12,084 & 11,502 & \\
\hline Baume de Vallorgues & FR & Kn-61 & 10,910 & 85 & 13,065 & 12,609 & \\
\hline Baume de Vallorgues & FR & Hv-1344 & 10,910 & 225 & 13,302 & 12,390 & \\
\hline Baume de Vallorgues & FR & $\mathrm{KN}-1061$ & 11,030 & 85 & 13,111 & 12,685 & \\
\hline Baume de Vallorgues & FR & Kn-68 & 11,200 & 115 & 13,310 & 12,765 & \\
\hline Baume de Vallorgues & FR & Hv-1345 & 11,270 & 230 & 13,601 & 12,667 & \\
\hline Bedburg-Königshoven & $\mathrm{DE}$ & $\mathrm{KN}-4137$ & 10,290 & 100 & 12,523 & 11,648 & \\
\hline Bedburg-Königshoven & $\mathrm{DE}$ & $\mathrm{KN}-4138$ & 10,670 & 100 & 12,839 & 12,230 & \\
\hline Bedburg-Königshoven & $\mathrm{DE}$ & $\mathrm{KN}-4136$ & 10,920 & 100 & 13,072 & 12,620 & \\
\hline Blot & FR & Ly-502 & 11,250 & 500 & 14,807 & 11,830 & \\
\hline Bois Ragot & FR & Gif-3580 & 10,990 & 160 & 13,189 & 12,599 & \\
\hline Bois-du-Cantet & FR & Ly-2614 & 10,920 & 140 & 13,106 & 12,590 & \\
\hline Brimfield & UK & BIRM-404 & 10,625 & 282 & 13,142 & 11,508 & \\
\hline Brohltal & $\mathrm{DE}$ & $?$ & 11,025 & 90 & 13,112 & 12,677 & \\
\hline Brohltal & $\mathrm{DE}$ & Hv-11774 & 11,075 & 185 & 13,293 & 12,626 & \\
\hline Brohltal & $\mathrm{DE}$ & $?$ & 11,085 & 90 & 13,166 & 12,701 & \\
\hline Brohltal & $\mathrm{DE}$ & Hd-17101 & 11,121 & 28 & 13,139 & 12,831 & \\
\hline Brohltal & $\mathrm{DE}$ & $\mathrm{KN}-3800$ & 11,240 & 100 & 13,341 & 12,857 & \\
\hline Brohltal & $\mathrm{DE}$ & KN-3801 & 11,260 & 95 & 13,342 & 12,894 & \\
\hline Budel IV & $\mathrm{NE}$ & GrN-1687 & 11,070 & 90 & 13,147 & 12,692 & \\
\hline Butzsee & $\mathrm{DE}$ & OxA-8743 & 10,185 & 65 & 12,121 & 11,502 & AMS \\
\hline Capeau (Lavalduc) & FR & HV-2126 & 11,700 & 450 & 15,061 & 12,680 & \\
\hline Champréveyres & $\mathrm{CH}$ & B-4530 & 11,120 & 110 & 13,243 & 12,714 & \\
\hline Chez Jugie & FR & Ly-1601 & 11,730 & 530 & 15,594 & 12,572 & \\
\hline Chez Jugie & FR & Ly-1572 & 11,840 & 580 & 16,068 & 12,617 & \\
\hline Closeau & FR & Ly-7190 & 10,465 & 110 & 12,614 & 12,034 & \\
\hline
\end{tabular}


Table 9 (continued)

\begin{tabular}{|c|c|c|c|c|c|c|c|}
\hline Site name & Country & Lab ID & Date & Uncertainty & From cal BP & To cal BP & Type \\
\hline Closeau & FR & Ly-7190 & 10,470 & 110 & 12,615 & 12,039 & \\
\hline Closeau & FR & Ly-7189 & 10,670 & 110 & 12,875 & 12,221 & \\
\hline Closeau & FR & Ly-7189 & 10,670 & 110 & 12,875 & 12,221 & \\
\hline Colombier & FR & Ly-4809 & 11,080 & 250 & 13,451 & 12,551 & \\
\hline Colombier & FR & Ly-4810 & 11,230 & 420 & 14,091 & 12,104 & \\
\hline Colombier & FR & Gif-8717 & 11,460 & 310 & 13,965 & 12,680 & \\
\hline Combarelles & FR & Ly-3201 & 11,380 & 210 & 13,707 & 12,782 & \\
\hline Conty & FR & Ly-6999 & 11,080 & 60 & 13,125 & 12,738 & \\
\hline Conty & FR & Ly-7407 & 11,130 & 80 & 13,212 & 12,747 & \\
\hline Cornille & FR & Ly-427 & 10,870 & 320 & 13,440 & 11,833 & \\
\hline Cornille & FR & Ly-449 & 10,920 & 210 & 13,275 & 12,425 & \\
\hline Coufin & FR & Ly-2931 & 11,020 & 150 & 13,201 & 12,622 & \\
\hline Courbet & FR & BM-303 & 11,110 & 160 & 13,285 & 12,665 & \\
\hline Cuze de Neussargues & FR & Ly-3261 & 11,580 & 300 & 14,127 & 12,757 & \\
\hline Dinslaken & $\mathrm{DE}$ & & 10,750 & 105 & 12,913 & 12,431 & \\
\hline Dinslaken & $\mathrm{NE}$ & $\mathrm{Hv}-1414$ & 10,790 & 105 & 12,942 & 12,543 & \\
\hline Dufaure & FR & Ly-2666 & 10,910 & 220 & 13,288 & 12,404 & \\
\hline Dumas (Saut du Loup) & FR & Ly-320 & 11,500 & 380 & 14,500 & 12,597 & \\
\hline Duruthy & FR & Ly-858 & 11,150 & 220 & 13,416 & 12,621 & \\
\hline Duurswoude II & $\mathrm{NE}$ & GrN-1565 & 11,090 & 90 & 13,171 & 12,705 & \\
\hline Duurswoude Oud Leger & $\mathrm{NE}$ & GrN-607 & 10,800 & 250 & 13,245 & 12,072 & \\
\hline Duurswoude Oud Leger & $\mathrm{NE}$ & GrN-4871 & 11,150 & 190 & 13,362 & 12,653 & \\
\hline Een-Schipsloot pit I & $\mathrm{NE}$ & GrN-6341 & 10,495 & 60 & 12,595 & 12,143 & \\
\hline Een-Schipsloot pit I & $\mathrm{NE}$ & Y-139-2 & 10,880 & 160 & 13,134 & 12,544 & \\
\hline Eglises & FR & Gif-1434 & 11,800 & 500 & 15,507 & 12,645 & \\
\hline Espelugues, Le Calvaire CPE Gauche & FR & Ly-1905 & 11,750 & 430 & 15,068 & 12,750 & \\
\hline Felsstalle & $\mathrm{DE}$ & $\mathrm{H}-7442-7491$ & 11,285 & 246 & 13,650 & 12,664 & \\
\hline Ferrassie & FR & Gif-4263 & 11,150 & 120 & 13,278 & 12,727 & \\
\hline Fontbregoua & FR & Gif-2994 & 11,200 & 150 & 13,351 & 12,716 & \\
\hline Gare de Couze & FR & BM-1615 & 11,230 & 180 & 13,422 & 12,695 & \\
\hline Gazel & FR & Gif-2654 & 10,760 & 190 & 13,104 & 12,145 & \\
\hline Geldrop I & $\mathrm{NE}$ & GrN-1059 & 10,960 & 85 & 13,081 & 12,653 & \\
\hline Geldrop I & $\mathrm{NE}$ & GrN-603 & 11,020 & 230 & 13,367 & 12,549 & \\
\hline Gerbaix “dessus” & FR & Ly-1952 & 10,670 & 170 & 12,949 & 12,069 & \\
\hline Gigot & FR & Lv-1111 & 10,980 & 80 & 13,086 & 12,666 & \\
\hline Gigot & FR & Lv-1112 & 10,980 & 80 & 13,086 & 12,666 & \\
\hline Gigot & FR & Lv-1110 & 11,110 & 95 & 13,206 & 12,714 & \\
\hline Gönnersdorf & $\mathrm{DE}$ & KN-1979 & 10,540 & 210 & 12,905 & 11,711 & \\
\hline Goutte Roffat & FR & Ly-2150 & 10,860 & 210 & 13,260 & 12,223 & \\
\hline Gramsbergen II & $\mathrm{NE}$ & GrN-8074 & 11,130 & 60 & 13,177 & 12,773 & \\
\hline Grotte du Renne, Arcy-sur-Cure & FR & BM-1818 & 10,500 & 190 & 12,775 & 11,708 & \\
\hline Grotte du Renne, Arcy-sur-Cure & FR & L-340B & 10,900 & 250 & 13,316 & 12,155 & \\
\hline Grotte du Renne, Arcy-sur-Cure & FR & $\mathrm{L}-340 \mathrm{~A}$ & 11,400 & 250 & 13,775 & 12,738 & \\
\hline Horn-Haelen & $\mathrm{NE}$ & GrN-498 & 10,950 & 300 & 13,436 & 12,110 & \\
\hline Horn-Haelen & $\mathrm{NE}$ & GrN-497 & 11,000 & 320 & 13,575 & 12,123 & \\
\hline Horn-Haelen & $\mathrm{NE}$ & GrN-7297 & 11,200 & 100 & 13,299 & 12,793 & \\
\hline Jean-Pierre I & FR & Ly-1190 & 10,620 & 210 & 13,068 & 11,827 & \\
\hline Jean-Pierre I & FR & Ly-596 & 10,750 & 300 & 13,289 & 11,769 & \\
\hline Jean-Pierre I & FR & Ly-626 & 11,340 & 260 & 13,732 & 12,688 & \\
\hline Jean-Pierre II & FR & Ly-625 & 10,470 & 200 & 12,758 & 11,508 & \\
\hline Jean-Pierre II & FR & Ly-692 & 11,590 & 330 & 14,465 & 12,679 & \\
\hline Kastelhöhle & $\mathrm{CH}$ & B-4254 & 11,320 & 130 & 13,450 & 12,884 & \\
\hline Kesslerloch & $\mathrm{CH}$ & B3327 & 11,220 & 120 & 13,333 & 12,773 & \\
\hline Kniegrotte & $\mathrm{DE}$ & GrN-6649 & 10,230 & 90 & 12,393 & 11,502 & \\
\hline La Colombière & FR & W-150 & 11,750 & 600 & 16,125 & 12,551 & \\
\hline La Fru & FR & Ly-4326 & 10,360 & 150 & 12,603 & 11,625 & \\
\hline La Fru & FR & Ly-2916 & 10,750 & 190 & 13,097 & 12,143 & \\
\hline La Fru & FR & Ly-3596 & 10,800 & 230 & 13,189 & 12,105 & \\
\hline La Fru & FR & Ly-3594 & 10,990 & 190 & 13,252 & 12,583 & \\
\hline La Fru & FR & Ly-2912 & 11,030 & 250 & 13,423 & 12,433 & \\
\hline La Fru & FR & Ly-2917 & 11,030 & 250 & 13,423 & 12,433 & \\
\hline La Fru & FR & Ly-2499 & 11,190 & 210 & 13,436 & 12,646 & \\
\hline La Fru & FR & Ly-3595 & 11,240 & 160 & 13,405 & 12,731 & \\
\hline La Garenne Grand abri & FR & Gif-34 & 11,230 & 500 & 14,606 & 11,815 & \\
\hline La Garenne-Blanchard & FR & C-577 & 11,109 & 480 & 14,159 & 11,624 & \\
\hline La Garenne-Blanchard & FR & Lv-383 & 11,280 & 220 & 13,592 & 12,681 & \\
\hline Lavalduc, Valduc & FR & $\mathrm{Hv}-?$ & 11,690 & 450 & 15,050 & 12,675 & \\
\hline Le Brassot & FR & Ly-9015 & 11,090 & 95 & 13,179 & 12,698 & \\
\hline Le Morin & FR & Gif-2105 & 10,480 & 200 & 12,770 & 11,615 & \\
\hline Lemforde & $\mathrm{DE}$ & Hv-14972 & 10,955 & 315 & 13,480 & 12,065 & \\
\hline Les Freydières & FR & Ly-451 & 11,380 & 180 & 13,675 & 12,851 & \\
\hline Lespaux & FR & Ly-3308 & 10,580 & 210 & 12,936 & 11,765 & \\
\hline Magdeleine & FR & Y-1109 & 11,180 & 300 & 13,708 & 12,563 & \\
\hline Margot & FR & Lv-1242 & 11,110 & 120 & 13,244 & 12,698 & \\
\hline Mehlbeck, Friedhof & $\mathrm{DE}$ & Hv-17306 & 10,515 & 95 & 12,620 & 12,110 & \\
\hline
\end{tabular}


Table 9 (continued)

\begin{tabular}{|c|c|c|c|c|c|c|c|}
\hline Site name & Country & Lab ID & Date & Uncertainty & From cal BP & To cal BP & Type \\
\hline Meiendorf & $\mathrm{DE}$ & Y-158 & 10,760 & 250 & 13,214 & 11,998 & \\
\hline Meiendorf D & $\mathrm{DE}$ & Y-158-2 & 10,760 & 250 & 13,214 & 11,998 & \\
\hline Messingham & UK & Birm-349 & 10,280 & 120 & 12,540 & 11,502 & \\
\hline Michelberg & $\mathrm{DE}$ & Bonn-763 & 10,800 & 100 & 12,926 & 12,554 & \\
\hline Miesenheim 2 & $\mathrm{DE}$ & Zürich & 10,840 & 195 & 13,208 & 12,224 & \\
\hline Miesenheim 2 & $\mathrm{DE}$ & KN-3519 & 11,040 & 220 & 13,337 & 12,572 & \\
\hline Miesenheim 2 & $\mathrm{DE}$ & KN-3520 & 11,070 & 100 & 13,163 & 12,683 & \\
\hline Miesenheim 2 & $\mathrm{DE}$ & $\mathrm{KN}-3518$ & 11,080 & 220 & 13,361 & 12,591 & \\
\hline Miesenheim 2 & $\mathrm{DE}$ & KN-3576 & 11,160 & 95 & 13,266 & 12,766 & \\
\hline Miesenheim 2 & $\mathrm{DE}$ & $\mathrm{KN}-3516$ & 11,230 & 95 & 13,326 & 12,856 & \\
\hline Miesenheim 2 & $\mathrm{DE}$ & KN-3579 & 11,240 & 95 & 13,332 & 12,871 & \\
\hline Milheeze Hutseberg, Milheeze Ia & $\mathrm{NE}$ & GrN-2314 & 10,880 & 125 & 13,076 & 12,586 & \\
\hline Milheeze-Hogeloop & NE & GrN-16508 & 10,810 & 60 & 12,870 & 12,581 & \\
\hline Moosbuhl & $\mathrm{CH}$ & B-2313 & 11,180 & 120 & 13,299 & 12,745 & \\
\hline Mother Grundy's Parlour & UK & Q-1459 & 11,160 & 170 & 13,337 & 12,673 & \\
\hline Mother Grundy's Parlour & UK & Q-1483 & 11,285 & 180 & 13,485 & 12,710 & \\
\hline Mother Grundy's Parlour & UK & Q-1484 & 11,320 & 230 & 13,660 & 12,699 & \\
\hline Moulin a Troubat & FR & Ly-5275 & 10,765 & 96 & 12,904 & 12,545 & \\
\hline Moulin a Troubat & FR & & 11,320 & 410 & 14,223 & 12,218 & \\
\hline Moulin-du-Roc & FR & Ly-5444 & 11,340 & 170 & 13,585 & 12,797 & \\
\hline Nahe LA 11 & $\mathrm{DE}$ & & 10,172 & 45 & 12,046 & 11,643 & \\
\hline Nahe LA 11 & $\mathrm{DE}$ & & 10,544 & 49 & 12,620 & 12,393 & \\
\hline Nahe LA 11 & $\mathrm{DE}$ & & 10,610 & 80 & 12,713 & 12,225 & \\
\hline Nettetal & $\mathrm{DE}$ & W-525 & 10,800 & 300 & 13,336 & 11,826 & \\
\hline Nettetal & $\mathrm{DE}$ & ? & 10,880 & 95 & 13,061 & 12,587 & \\
\hline Nettetal & $\mathrm{DE}$ & $?$ & 10,950 & 190 & 13,241 & 12,560 & \\
\hline Oldeholtwolde & $\mathrm{NE}$ & GrN-12280 & 11,080 & 280 & 13,581 & 12,427 & \\
\hline Oldeholtwolde & $\mathrm{NE}$ & GrN-10274 & 11,540 & 270 & 13,995 & 12,831 & \\
\hline Ossom's Cave & UK & GrN-7400 & 10,590 & 70 & 12,681 & 12,230 & \\
\hline Oudehaske & $\mathrm{NE}$ & GrN-18783 & 11,120 & 70 & 13,180 & 12,750 & \\
\hline Pech-Merle & FR & Ly-1200 & 11,460 & 390 & 14,500 & 12,570 & \\
\hline Pégourié & FR & Ly-3933 & 10,700 & 290 & 13,252 & 11,720 & \\
\hline Pégourié & FR & Ly-1390 & 11,290 & 320 & 13,812 & 12,593 & \\
\hline Pégourié & FR & Ly-1391 & 11,680 & 330 & 14,582 & 12,781 & \\
\hline Pincevent, La Grande-Paroisse & FR & GrN-4383 & 10,760 & 60 & 12,806 & 12,557 & \\
\hline Pincevent, La Grande-Paroisse & FR & GrN-4383 & 10,760 & 60 & 12,806 & 12,557 & \\
\hline Pincevent, La Grande-Paroisse & FR & Lv-293 & 11,310 & 330 & 13,860 & 12,587 & \\
\hline Pincevent, La Grande-Paroisse & FR & Lv-292 & 11,610 & 400 & 14,840 & 12,660 & \\
\hline Poeymau & FR & Ly-1789 & 10,700 & 290 & 13,252 & 11,720 & \\
\hline Pré de la Chapelle & FR & ETH-15760 & 11,230 & 90 & 13,318 & 12,870 & AMS \\
\hline Querenstede & $\mathrm{DE}$ & KN-1053 & 11,140 & 240 & 13,454 & 12,590 & \\
\hline Remouchamps & $\mathrm{BE}$ & Lv-535 & 10,380 & 170 & 12,636 & 11,506 & \\
\hline Rissen $14 a$ & $\mathrm{NE}$ & Y-157-A & 10,560 & 200 & 12,897 & 11,770 & \\
\hline Robin Hood's Cave & UK & BM-0603 & 10,390 & 90 & 12,560 & 11,984 & \\
\hline Robin Hood's Cave & UK & BM-603 & 10,390 & 90 & 12,560 & 11,984 & \\
\hline Robin Hood's Cave & UK & BM-0604 & 10,590 & 90 & 12,692 & 12,151 & \\
\hline Roc-aux-Sorciers, Abri Bourdois & FR & GrN-2912 & 10,800 & 120 & 13,065 & 12,534 & \\
\hline Roc-aux-Sorciers, Abri Bourdois & FR & GrN-2913 & 11,265 & 130 & 13,398 & 12,808 & \\
\hline Roc-aux-Sorciers, Abri Bourdois & FR & GrN-2916 & 11,265 & 130 & 13,398 & 12,808 & \\
\hline Rochedane & FR & Ly-1194 & 10,730 & 190 & 13,086 & 12,131 & \\
\hline Rochedane & FR & Ly-1193 & 11,060 & 470 & 14,039 & 11,620 & \\
\hline Rochedane & FR & Ly-1192 & 11,090 & 200 & 13,325 & 12,615 & \\
\hline Roquemissou Abri $\mathrm{n}^{\circ} 1$ des Usclades & FR & Gif-8438 & 10,250 & 80 & 12,388 & 11,648 & \\
\hline Sainte Eulalie & FR & Gif-1697 & 10,830 & 200 & 13,196 & 12,219 & \\
\hline Saleux 114 & FR & Gif-8706 & 10,640 & 90 & 12,757 & 12,225 & \\
\hline Salpêtrière & FR & Ly-937/ Ly-21678 & 10,680 & 300 & 13,245 & 11,627 & \\
\hline Salpêtrière & FR & Ly-938 & 11,080 & 250 & 13,451 & 12,551 & \\
\hline Sanglier & FR & Ly-6161 & 11,102 & 102 & 13,207 & 12,701 & \\
\hline Sanglier & FR & Ly-7286 & 11,180 & 80 & 13,276 & 12,810 & \\
\hline Seamer K & UK & CAR-841 & 10,960 & 110 & 13,100 & 12,634 & \\
\hline Solutré, L-13 & FR & Ly-315 & 10,900 & 400 & 13,696 & 11,649 & \\
\hline Soppensee & $\mathrm{CH}$ & ETH-5290 & 10,760 & 105 & 12,921 & 12,436 & AMS \\
\hline Soppensee & $\mathrm{CH}$ & ETH-1610 & 11,180 & 100 & 13,284 & 12,775 & AMS \\
\hline St. Bees & UK & BIRM-1144 & 10,430 & 165 & 12,660 & 11,715 & \\
\hline St. Bees & UK & BIRM-649 & 11,180 & 120 & 13,299 & 12,745 & \\
\hline St. Bees & UK & BIRM-648 & 11,300 & 220 & 13,617 & 12,692 & \\
\hline Sun Hole & UK & Birm-820 & 10,280 & 120 & 12,540 & 11,502 & \\
\hline Sun Hole & UK & Birm-821 & 10,470 & 190 & 12,734 & 11,626 & \\
\hline Tai & FR & Ly-4216 & 11,270 & 190 & 13,486 & 12,688 & \\
\hline Thur & $\mathrm{DE}$ & $\mathrm{KN}-2868$ & 11,050 & 120 & 13,176 & 12,658 & \\
\hline Thur & $\mathrm{DE}$ & $\mathrm{KN}-2869$ & 11,110 & 90 & 13,199 & 12,718 & \\
\hline Thur & $\mathrm{DE}$ & $\mathrm{KN}-2870$ & 11,250 & 95 & 13,336 & 12,884 & \\
\hline Tilloy, Moque Panier & FR & Gif-5838 & 10,400 & 180 & 12,661 & 11,502 & \\
\hline Trollesgave & DK & $\mathrm{K}-2641$ & 11,070 & 120 & 13,198 & 12,669 & \\
\hline Trollesgave & DK & K-2509 & 11,100 & 160 & 13,280 & 12,660 & \\
\hline Trou du Frontal & $\mathrm{BE}$ & Lv-1135 & 10,720 & 120 & 12,929 & 12,390 & \\
\hline
\end{tabular}


Table 9 (continued)

\begin{tabular}{|c|c|c|c|c|c|c|c|}
\hline Site name & Country & Lab ID & Date & Uncertainty & From cal BP & To cal BP & Type \\
\hline Usselo & $\mathrm{NE}$ & Y-139-2 & 10,880 & 160 & 13,134 & 12,544 & \\
\hline Usselo & NE & Y-139-3 & 11,350 & 150 & 13,571 & 12,872 & \\
\hline Vailly-sur-Aisne & FR & SA-53 & 11,550 & 450 & 14,912 & 12,595 & \\
\hline Venerolles (la Sabliere) & FR & Gif-8101 & 10,510 & 110 & 12,634 & 12,076 & \\
\hline Verberie, Le Buisson Campin & FR & Ly-3404 & 10,640 & 180 & 12,940 & 12,003 & \\
\hline Weissensee & $\mathrm{DE}$ & KN-3624 & 11,400 & 230 & 13,751 & 12,771 & \\
\hline Wustermark 22 & $\mathrm{DE}$ & $?$ & 10,370 & 75 & 12,529 & 11,982 & \\
\hline Zigeunerfels & $\mathrm{DE}$ & KN-3485 & 10,340 & 90 & 12,529 & 11,824 & \\
\hline Zigeunerfels & $\mathrm{DE}$ & $\mathrm{KN}-3488$ & 10,400 & 90 & 12,561 & 11,997 & \\
\hline Zigeunerfels & $\mathrm{DE}$ & KN-3784 & 10,500 & 110 & 12,628 & 12,068 & \\
\hline Zigeunerfels & $\mathrm{DE}$ & $\mathrm{KN}-3483$ & 10,650 & 110 & 12,799 & 12,151 & \\
\hline Zigeunerfels & $\mathrm{DE}$ & KN-3477 & 10,880 & 120 & 13,070 & 12,588 & \\
\hline Zigeunerfels & $\mathrm{DE}$ & $\mathrm{KN}-3476$ & 11,070 & 95 & 13,155 & 12,688 & \\
\hline Zigeunerfels & $\mathrm{DE}$ & KN-3475 & 11,180 & 100 & 13,284 & 12,775 & \\
\hline Zigeunerfels & $\mathrm{DE}$ & KN-3487 & 11,220 & 120 & 13,333 & 12,773 & \\
\hline Zigeunerfels & $\mathrm{DE}$ & $\mathrm{KN}-3480$ & 11,250 & 100 & 13,348 & 12,872 & \\
\hline
\end{tabular}

same western Belgian sites (Trou Da Somme, Chaleux, Bois Laiterie) (Miller and Noiret, 2009) (Fig. 6). In contrast, local flint sources in the Paris Basin were amply sufficient to provision these sites, and flint from Belgian sources is rare (Mauger, 1994; Féblot-Augustins, 1997; Djindjian, 2005). These data support the hypothesis of seasonal migration between the Paris Basin and at least western Belgium, indicating movement from southwest to northeast. Such movement may reflect following reindeer along their own migration route, the Paris Basin in fall-winter and Belgian territory during summer (see Rensink, 1993, among others). Flint from the Hesbaye Plateau in Belgium is the main lithic raw material in all Belgian Magdalenian sites, additionally supplying Rhineland sites.

Overall, the Magdalenian sites in northwest Europe vary in function and include large, multi-activity base camps, flint procurement sites, short-term hunting camps and extended stay seasonal occupations. Large, complex sites interpreted as base

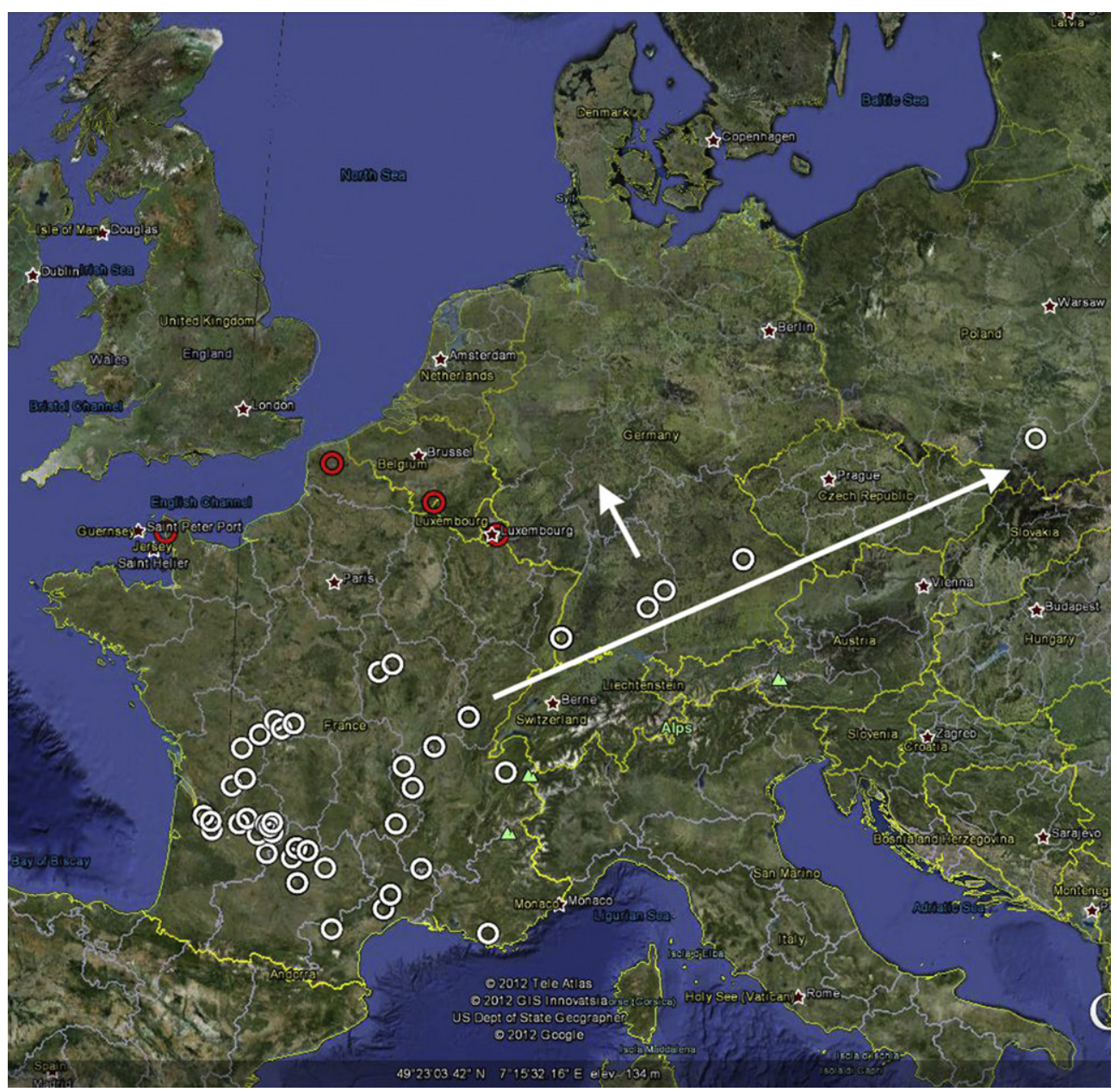

Fig. 3. Map showing the initial expansion trajectory of the Magdalenian during the $19-17.5 \mathrm{ka}$ cal BP phase. 


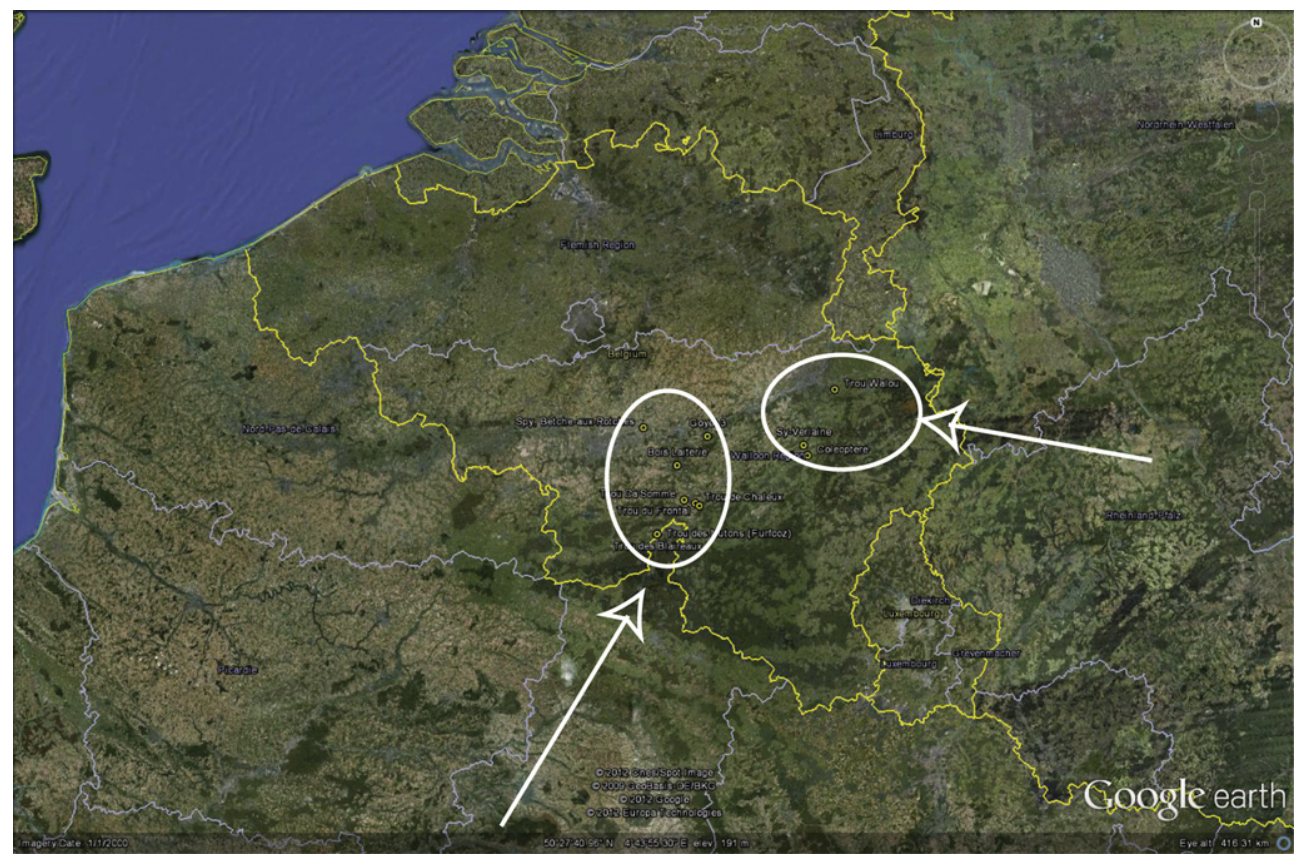

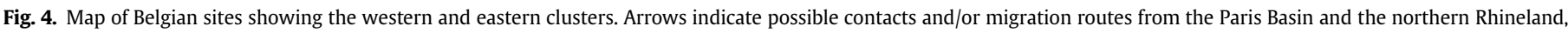
making Belgian territory part of the territories of each.

camps are present in the Paris Basin (Etiolles, Marsangy, Pincevent, Verberie, among others) and the Rhineland (Gönnersdorf), containing evidence for multiple activities (flintknapping, hideworking, stone processing, butchery, cooking) and features including hearths, dwelling structures, stone plaquette paving, mobile art (e.g., engraved plaquettes). In Belgium, the only comparable site is Chaleux, a cave site rather than open-air, but less elaborate than those in France and Germany. It is more likely an extended stay site, for the seasonal exploitation of the resources in the Belgian territory (flint on the Hesbaye Plateau, subsistence) and linked with the larger base camps in the Paris Basin. Several small sites around Chaleux (Trou Da Somme, Frontal, Nutons) can be interpreted as short-term hunting sites, as well as Bois Laiterie, several km downstream along the Meuse and en route toward the Hesbaye Plateau. The open-air sites of Orp (Vermeersch and Vynkier, 1980; Vermeersch et al., 1987) and Kanne (Vermeersch et al., 1985), both located at sources of Cretaceous flint, are interpreted as flint procurement workshops, possibly embedded in subsistence activities, or vice versa. The flint from Orp (or other potential comparable sites on the Hesbaye Plateau) was used at Belgian sites, and apparently not transported to the Paris Basin. In contrast, flint from the Meuse terraces and/or sites such as Kanne, near Maastricht, was transported to northern Rhineland sites. The eastern Belgian sites (Walou, Sy Verlaine) may have been part of the greater Rhineland territory, possibly hunting sites.

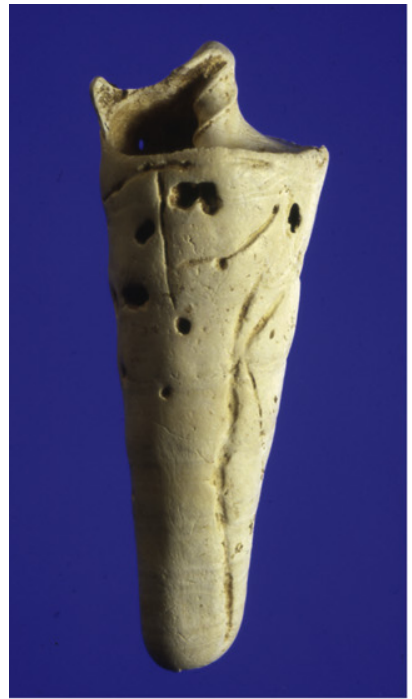

1

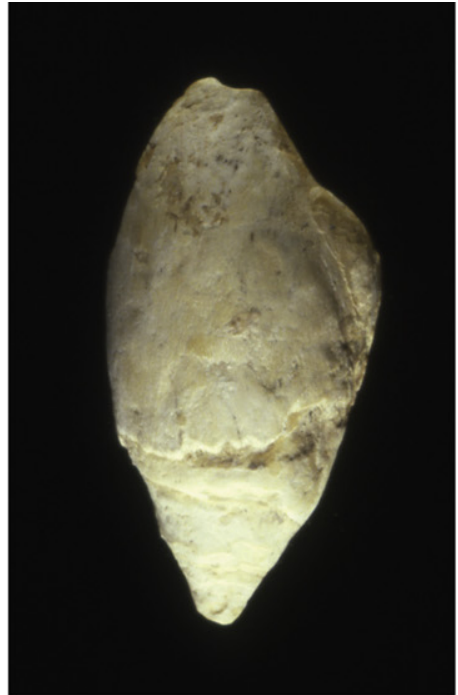

2

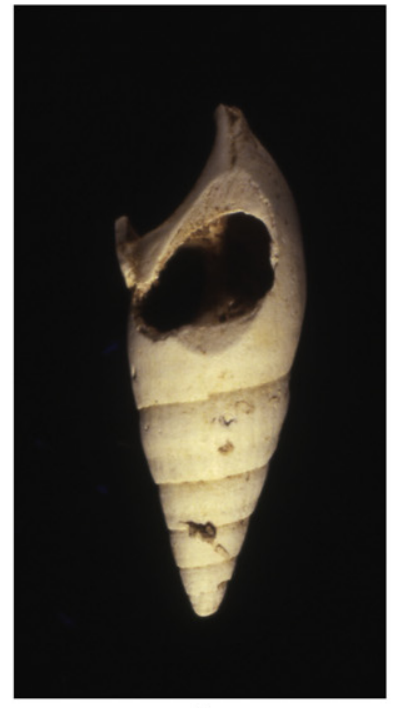

3

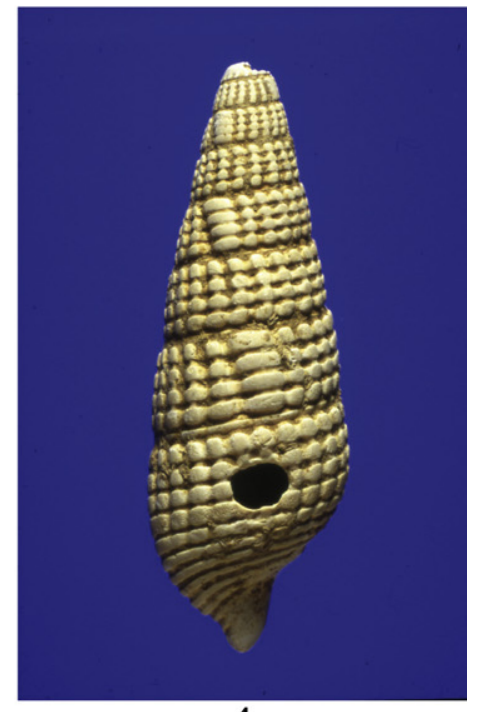

4

Fig. 5. Fossil marine shells present at Belgian sites. 1-3: Bois Laiterie, 4: Chaleux. 


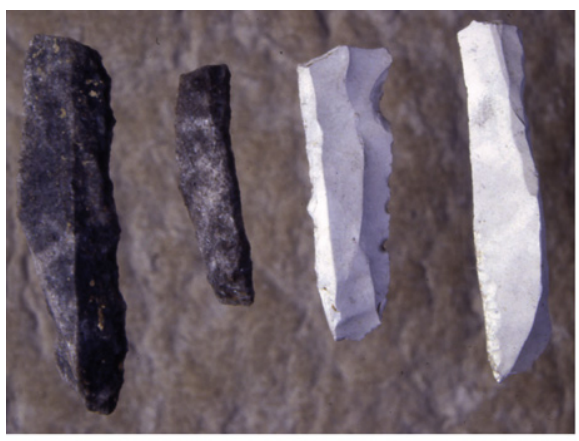

1

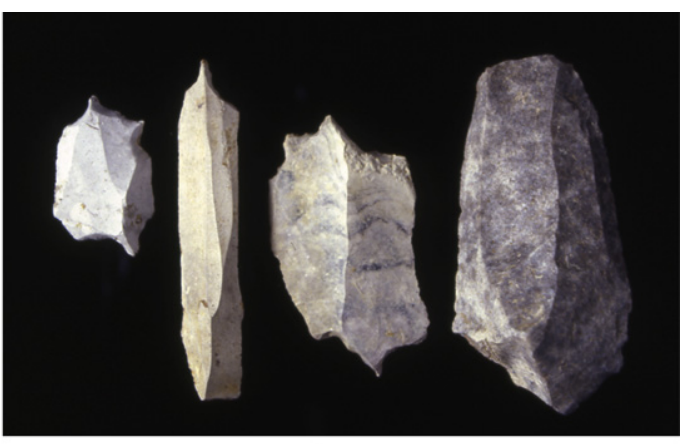

2

Fig. 6. Examples of silicified limestone and flint from Trou Da Somme. 1: blades, 2: perçoirs and an endscraper.

Interestingly, even small sites interpreted as short-term hunting stations have yielded mobile art and fossil marine shells: an engraved plaquette at Trou Da Somme, several shells at Bois Laiterie, one from Trou Da Somme and shells at Sy Verlaine. Chaleux, a larger site, contains several engraved plaquettes and shells.

\section{Conclusions}

Both demographic and climatic factors played significant roles in the expansion of the Magdalenian to Northwest Europe. Indeed, climate played a limiting role in the occupation of northern Europe throughout the Paleolithic: Neandertals are present during warm phases (Seclin, Scladina, Rocourt, Wallertheim; Spy at 36,000 BP) (see e.g., Finlayson and Carrión, 2007), while modern humans become locally extinct or abandon the northern latitudes at the onset of the LGM. Even during the Early Upper Paleolithic, the Aurignacian appears to be correlated with warm oscillations in the north. The Magdalenian does not appear in northwest Europe until the warming trend prior to $14.67 \mathrm{ka}$ cal BP. Dated sites are present as far north as Wales and Scotland and northern Germany during the Meiendorf and Bølling, becoming more widespread in England and present in northern Denmark during the Allerød. New technocomplexes appear during GI-1e (Hamburgian), followed later by the Creswellian, Federmesser, Ahrensburgian and Brommean in the more northern regions (Great Britain, northern Germany, Denmark). The cold event of the Younger Dryas (GS-1), nearly 1000 years, appears to have been a factor in the southward movement of these technocomplexes, which remained, however, in northwest Europe, replacing the Magdalenien.

Finally, this study has shown that critical evaluation of radiocarbon dates, particularly conventional dates, is necessary when examining the chronological and geographic distribution of Lateglacial sites. For this paper, dates for all sites above $47^{\circ} \mathrm{N}$ latitude were evaluated; southern French sites were beyond the scope of this paper, their overall distribution generally reflecting Lateglacial occupation prior to expansion. However, researchers in this region for this period clearly take the validity of radiocarbon dates into consideration. Dates were excluded on the basis of large error ranges, lack of association with artifacts, dating done in the 1960s and 1970s, or evidence of contamination. The Radiocarbon Palaeolithic Database is a key tool, but the data within it needs to be evaluated, with dates to be rejected clearly identified and reason for exclusion indicated. Finally, a significant number of dates obtained in the last decade are lacking from the database; it is hoped that researchers will send their results to the database project so that it reflects the latest advances in Palaeolithic chronological research.

\section{Acknowledgments}

I would like to thank Lawrence Straus, Thomas Terberger and Denise Leesch for their detailed comments on this paper. Any errors that remain are, however, mine.

\section{Appendix A. Supplementary data}

Supplementary data associated with this article can be found, in the online version, at doi:10.1016/j.quaint.2012.05.034.

\section{References}

Alley, R.B., Marotzke, J., Nordhaus, W.D., Overpeck, J.T., Peteet, D.M., Pielke, R.A., Pierrehumbert, R.T., Rhines, P.B., Stocker, T.F., Talley, L.D., Wallace, J.M., 2003. Abrupt climate change. Science 299, 2005-2010.

Alley, R.B., Clark, P.U., 1999. The deglaciation of the northern hemisphere: a global perspective. Annual Reviews of Earth and Planetary Sciences 27, 149-182.

Björck, S., Rundgren, M., Ingolfsson, O., Funder, S., 1997. The Preboreal oscillation around the Nordic Seas: terrestrial and lacustrine responses. Journal of Quaternary Science 12 (6), 455-465.

Björck, S., Walker, M., Cwynar, L., Johnsen, S., Knudsen, K.-L., Lowe, J.-J., Wohlfarth, B., INTIMATE members, 1998. An event stratigraphy for the Last Termination in the North Atlantic region based on the Greenland ice-core record: a proposal by the INTIMATE group. Journal of Quaternary Science 13, 283-292.

Blockley, S.P.E., Donahue, R.E., Pollard, A.M., 2000. Radiocarbon calibration and Late Glacial occupation in Northwest Europe. Antiquity 74, 112-119.

Bodu, P., Mevel, L., 2008. Enquête autour des lames tranchantes de l'Azilien ancien. Le cas du niveau inférieur du Closeau (Rueil-Malmaison, Hauts-de-Seine, France). L'Anthropologie 112, 509-543.

Brauer, A., Haug, G., Dulski, P., Sigman, D., Negendank, J., 2008. An abrupt wind shift in western Europe at the onset of the Younger Dryas cold period. Nature Geoscience 1, 520-523.

Cattelain, P., 2005. Propulseurs magdaléniens: marquers culturels régionaux? In: Dujardin, V. (Ed.), Industries osseuses et parures du Solutréen au Magdalénien en Europe Société préhistoriques française, Mémoires 34, pp. 301-317.

Charles, R. 1994. Food for Thought: Late Magdalenian chronology and faunal exploitation in the north-western Ardennes. Oxford University, doctoral thesis.

Charles, R., 1996. Back into the North: the radiocarbon evidence for the human recolonisation of the North Western Ardennes after the Last Glacial Maximum. Proceedings of the Prehistoric Society 62, 1-17.

Charles, R., 1998. Late Magdalenian Chronology and Faunal Exploitation in the North-Western Ardennes. In: Oxford, BAR International Series 737.

Conneller, C., 2007. Inhabiting new landscapes: settlement and mobility in Britain after the Last Glacial Maximum. Oxford Journal of Archaeology 26, 215-237.

Clark, P., Dyke, A., Shakun, J., Carlson, A., Clark, J., Wohlfarth, B., Mitrovica, J., Hostetler, S., McCabe, M., 2009. The Last Glacial Maximum. Science 325, 710-714.

Coope, G.R., Joachim, M.J., 1980. Lateglacial environmental changes interpreted from fossil coleopteran from St Bees, Cumbria, NW England. In: Lowe, J.J., Gray, J.M., Robinson, J.E. (Eds.), Studies in the Lateglacial of North-West Europe. Pergamon Press, Oxford, pp. 55-68.

De Bie, M., Caspar, J.-P., 1994. Rekem, a Federmesser Camp on the Meuse River Bank. In: Instituut voor het Archaeologisch Patrimonium, vol. 1. Leuven University Press, Leuven.

Delibrias, G., Guillier, M.-Th., Labeyrie, J., 1982. GIF natural radiocarbon measurements ix. Radiocarbon 24 (3), 291-343. 
Dewez, M., 1987. Le Paléolithique supérieur récent dans les Grottes de Belgique. In: Publications d'Histoire de l'Art et d'Archéologie de l'Université Catholique de Louvain, LVII. Louvain-la-Neuve.

Djindjian, F., 2005. Approvisionnement en matières premières dans le Paléolithique supérieur de l'Europe occidentale: Méthodes et résultats. Archeometriai Mühely 4, 1-16.

Donnelly, J., Driscoll, N., Uchupi, E., Keigwan, L., Schwab, W., Thieler, R., Swift, S., 2005. Catastrophic meltwater discharge down the Hudson Valley: a potential trigger for the Intra-Allerød cold period. Geology 33, 89-92.

Fagnard, J.-P., 1988. Le gisement Levert à Hallines (Pas-de-Calais). Révue archéologique de Picardie, numéro spécial 7, pp. 34-45.

Féblot-Augustins, J., 1997. La circulation des matières premières au Paléolithique. ERAUL 75. Liège.

Finlayson, C., Carrión, J., 2007. Rapid ecological turnover and its impact on Neanderthal and other human populations. Trends in Ecology and Evolution 22 (4), 213-222.

Housley, R.A., Gamble, C.S., Street, M., Pettitt, P., 1997. Radiocarbon evidence for the Lateglacial human recolonisation of Northern Europe. Proceedings of the Prehistoric Society 63, 25-54.

Housley, R.A., Gamble, C.S., Pettitt, P., 2000. Reply to Blockley, Donahue and Pollard. Antiquity 74, 119-121.

Jacobi, R., Higham, T., 2011. The Later Upper Palaeolithic recolonisation of Britain: new results from AMS radiocarbon dating In: Ashton, N., Lewis, S.G., Stringer C (Eds.), The Ancient Human Occupation of Britain. Developments in Quaternary Science 14. Elsevier, London, pp. 223-247.

Jochim, M., Herhain, C., Starr, H., 1999. The Magdalenian colonization of Southern Germany. American Anthropologist 101, 129-142.

Kozłowski, J.K., 2001. La recolonisation Tardiglaciaire et les changements culturels à la limite Pléistocène-Holocène sur la Grande Plaine. In: Otte, M. (Ed.), Préhistoire de la Grande Plaine du Nord de l'Europe. Actes du Colloque Chaire Francqui interuniversitaire au titre étranger. ERAUL 99. Université de Liège, Liège, pp. 115-127.

Kozłowski, S.K., Połtowicz-Bobak, M., Bobak, D., Terberger, T., 2012. New information from Maszycka Cave and the Late Glacial recolonisation of Central Europe. Quaternary International 272-273, 288-296.

Leesch, D., Müller, W., Nielsen, E., Bullinger, J., 2012. The Magdalenian in Switzerland: re-colonization of a newly accessible landscape. Quaternary International 272-273, 191-208.

Litt, T., Brauer, A., Goslar, T., Merkt, J., Balaga, K., Müller, H., RalskaJasiewiczowa, M., Stebich, M., Negendank, J.F.W., 2001. Correlation and synchronisation of Lateglacial continental sequences in northern central Europe based on annually-laminated lacustrine sediments. Quaternary Science Reviews 20, 1233-1249.

Liu, Z. Otto-Bliesner, B., He, F., Brady, E., Tomas, R., Clark, P., Carlson, A., LynchStieglitz, J., Curry, W., Brook, E., Erickson, D., Jacob, R., Kutzbach, J., Cheng, J., 2009. Transient Simulation of last deglaciation with a new mechanism for Bølling-Allerød warming. Science 325, 310-314.

Lozouet, P., Gautier, A., 1997. Coquillages fossiles et restes de "briquet" dans la Grotte du Bois Laiterie. In: Otte, M., Straus, L.G. (Eds.), La grotte du Bois Laiterie. Recolonisation magdalénienne de la Belgique, Liège: ERAUL 80 pp. 319-323.

Mauger, M., 1994. L'approvisionnement en matériaux siliceux au Paléolithique supérieur. In: Taborin, Y. (Ed.), Environnements et habitats magdaléniens dans le centre du Bassin Parisien. Paris, Maison des Sciences de l'Homme, Document d'Archéologie Française 43: 78-93. L'abri de La Fru (Savoie).

Miller, R., Noiret, P., 2009. Recent results for the Belgian Magdalenian. In: Street, M., Barton, N., Terberger, T. (Eds.), Humans, Environment and Chronology of the Late Glacial of the North European Plain, 15th Congress for the International Union of Pre- and Protohistoric Sciences (UISPP), 2-8 September 2006, Lisbon. Verlag des Römisch-germanischen Zentralmuseums Mainz, pp. 39-44.

Otte, M., Straus, L.G. (Eds.), 1997. La grotte du Bois Laiterie. Recolonisation magdalénienne de la Belgique, vol. 80. ERAUL, Liège.

Rensink, E. 1993. Moving into the North. Universiteit Leiden, doctoral thesis.

Rozoy, J., 1988. Le Magdalénien en Europe: démographie, groupes régionaux. Bulletin de la Société préhistorique luxembourgeoise 10, 139-158.

Sedlmeier, J., 2010. Die junepaläolithicischen Funde aus der mittleren Fundschicht der kastelhöhle in nordwestschweizerischen Kaltbrunnental (Gemeinde Himmelried SO). Ein nachweid für die Begehung der Schweiz durch Mensch und Tier kurz nach dem Kältemaximum der letzen Eiszeit. Jarbuch der Archäologie Schweiz 93, 7-34.

Severinghaus, J.P., Brook, E.J., 1999. Abrupt climate change at the end of the Last Glacial period inferred from trapped air in polar ice. Science 286, 930-934.

Severinghaus, J., Sowers, T., Brook, E., Alley, R., Bender, M., 1998. Timing of abrupt climate change at the end of the Younger Dryas interval from thermally fractionated gases in polar ice. Nature 391, 141-146.
Sima, A., Paul, A., Schulz, M., 2004. The Younger Dryas - an intrinsic feature of late Pleistocene climate change at millennial timescales. Earth and Planetary Science Letters 222, 741-750.

Shakun, J., Carlson, A., 2010. A global perspective on Last Glacial Maximum to Holocene climate change. Quaternary Science Reviews 29, 1801-1816.

Stevens, R., O'Connell, T., Hedges, R.E.M., Street, M., 2009. Radiocarbon and stable isotope investigations at the Central Rhineland sites of Gönnersdorf and Andernach-Martinsberg, Germany. Journal of Human Evolution 57 (2) 131-148.

Street, M., Terberger, T., 1999. The last Pleniglacial and the human settlement of Central Europe: new information from the Rhineland site of Wiesbaden-Igstadt Antiquity 73, 259-272.

Street, M., Terberger, T., 2004. The radiocarbon chronology of the German Upper Palaeolithic: fifteen years of cooperation with ORAU. In: Higham, T.F.G., BronkRamsey, C., Owen, D.C. (Eds.), Conference Proceedings, Radiocarbon and Archaeology, Fourth International Symposium, St; Catherine's College, Oxford, 9-14 April 2002. Oxford University School of Archaeology Monograph 62 pp. $281-302$

Street, M., Baales, M., Weninger, B., 1994. Absolute Chronologie des späten Paläolithikums und Frühmesolithikums im nördlich Rheinland. Archäologisches Korrespondenzblatt 24, 1-28.

Street, M., Barton, N., Terberger, T. (Eds.), 2009. Humans, Environment and Chronology of the Late Glacial of the North European Plain. 15th Congress for the International Union of Pre- and Protohistoric Sciences (UISPP), 2-8 September 2006, Lisbon. Verlag des Römisch-germanischen Zentralmuseums Mainz.

Street, M., Jöris, O., Turner, E., 2012. Magdalenian settlement in the German Rhineland - an update. Quaternary International 272-273, 231-250.

Taborin, Y., 1993. La parure en coquillage au Paléolithique. CNRS XXXIX, supplement to Gallia-Préhistoire, Paris.

Taborin, Y., 1994. Environnements et habitats magdaléniens dans le centre du Bassin parisien. Maison des Sciences de l'Homme, Paris.

Terberger, T., Street, M., 2003. New evidence for the chronology of the Aurignacian and the question of pleniglacial settlement in western Centra Europe. In: D'Errico, F., Zilhao, J. (Eds.), The Chronology of the Aurignacian and of the Transitional Technocomplexes, Dating, Stratigraphies, Cultura Implications. Proceedings of Symposium 6.1 of the XIVth Congress of the U.I.S.P.P. Liège, Belgium September 2001. Lisboa: Trabalhos de Arqueologia 33, pp. 213-221.

Terberger, T., Barton, N., Street, M., 2009. The Late Glacial reconsidered - recen progress and interpretations. In: Street, $M$., Barton, $N$., Terberger $T$. (Eds.) Humans, Environment and Chronology of the Late Glacial of the North European Plain, Tagungsbänder des Römisch-Germanischen Zentralmuseums Mainz 6, 189-207. Mainz and Bonn. (Workshop 14 for Commission XXXII, 15th U.I.S.P.P. Congress, Lisbon, September 2006), pp. 189-207.

Valentin B., 1995. Les groupes humains et leurs traditions au Tardiglaciaire dans le Bassin Parisien. Apports de la technologie lithique comparée. Université. Paris 1, doctoral thesis.

Vermeersch, P., 2011. Radiocarbon Palaeolithic Database, Version 12. Available at: http://ees.kuleuven.be/geography/projects/14c-palaeolithic/index.html.

Vermeersch, P., Leuwers, R., Van Peer, P., 1985. Un site magdalénien à Kanne. Archaeologica Belgica. NS 1, 17-54.

Vermeersch, P., Maes, M., 1996. Chronostratigraphy of the Magdalenian at Orp. Notae Praehistoricae 16, 87-90.

Vermeersch, P., Symens, N., 1988. Le Magdalénien de plein-air en Belgique. In: Otte, M. (Ed.), De la Loire à l'Oder. BAR S-444, Oxford, pp. 243-258.

Vermeersch, P., Symens, N., Vyncker, P., Gijelings, G., Lauwers, R., 1987. Orp, site Magdalénien de plein air (comm. de Orp-Jauche). Archaeologica Belgica 3, 7-56.

Vermeersch, P., Vynkier, P., 1980. Un site magdalénien à Orp. Archaeologica Belgica 223, 10-14

Weber, M.-J., Grimm, S., 2009. Dating the Hamburgian in the context of Lateglacial chronology. In: Crombé, Philippe, Van Strydonck, Mark, Sergant, Joris, Boudin, Mathieu, Bats, Machteld (Eds.), Chronology and Evolution Within the Mesolithic of North-West Europe: Proceedings of an International Meeting, Brussels, May 30th-June 1st 2007, Newcastle Upon Tyne: Cambridge Scholars Publishing, pp. 3-21.

Wenzel, S., 2005. Orp-East: indications for a dwelling. Notae Praehistoricae 25 , 83-90.

Williams, C., Flower, B., Hastings, D., Guilderson, T., Quinn, K., Goddard, E., 2010. Deglacial abrupt climate change in the Atlantic warm pool: a gulf of Mexico perspective. Paleoceanography 25 (PA4221), 1-12.

Ziesaire, P., 1994. Le Paléolithique supérieur du Grand-Duché de Luxembourg. Essai de synthèse. Bulletin de la Société Préhistorique Luxembourgeoise 16, 97-107. 